\title{
CONFORMAL FIELD THEORY and GEOMETRY of STRINGS
}

\author{
by \\ Jürg Fröhlich \\ Institut für Theoretische Physik, ETH-Hönggerberg, \\ 8093 Zürich, Switzerland \\ Krzysztof Gawędzki ${ }^{1}$ \\ C.N.R.S., Institut des Hautes Etudes Scientifiques, \\ 91440 Bures-sur-Yvette, France
}

\begin{abstract}
What is quantum geometry? This question is becoming a popular leitmotiv in theoretical physics and in mathematics. Conformal field theory may catch a glimpse of the right answer. We review global aspects of the geometry of conformal fields, such as duality and mirror symmetry, and interpret them within Connes' non-commutative geometry.
\end{abstract}

\section{Introduction}

Geometry has been used as a tool in classical physics in more interesting ways than in quantum physics. Analytic mechanics or Einstein's general relativity are outstanding examples of classical theories unseparable from their geometric content. It may seem then that it is enough to look at the quantized versions of analytic mechanics and of general relativity in order to understand how the quantum fluctuations modify geometry. In quantum mechanics, Poisson brackets become commutators and one could think that quantum symplectic geometry is the theory of canonical commutation relations and of their representations. This would be, however, too naive as the examples of difficulties with understanding quantum counterparts of the

\footnotetext{
${ }^{1}$ extended version of lectures given by the $2^{\text {nd }}$ author at the Mathematical Quantum Theory Conference held at the University of British Columbia, Vancouver, Canada, from August 4 to 8, 1993
} 
classical phenomena of integrability and chaos show. With general relativity, the situation is even worse: Einstein's gravity has resisted numerous attempts aimed at quantizing it. These unsuccessful efforts have convinced physicists that quantum Riemannian geometry should be rather different from the classical one, at least at very short distances. A possible picture of such a modified geometry emerges from string theory, the best available candidate for a consistent model of quantum gravity. The fundamental idea of string theory is to replace point-like objects by string-like (or loop-like) ones. This should deform the geometry based on the notion of a space of points. Quantum string theory is still rather poorly understood but may be studied, on the classical and perturbative level, by means of conformal (quantum, two-dimensional) field theory (CFT). Roughly speaking, models of CFT describe classical solutions of string theory. The perturbative expansion around the classical solutions is built by considering the CFT models on two-dimensional space-times of non-trivial topology. To understand the string geometry on the classical and perturbative level, one should then understand the (quantum) geometry of CFT's. Although a fully quantum string geometry at arbitrarily small scales may not be accessible to classical or perturbative analysis, its behavior in the large should be captured by the classical approximation. Below we shall concentrate on phenomena which distinguish the geometry in the large of CFT's from the conventional Riemann-Einstein geometry.

How should a physicist think about Riemannian geometry? As in other cases, she or he should extract general concepts from observable quantities. The trajectories of small bodies (test particles) are observable and, in general relativity, they are the time-like geodesics. The latter, when parametrized by the proper time, encode the complete information about the pseudo-Riemannian metric. The Lorentzian signature of the relevant geometry is, of course, the basic physical fact but, below, we shall limit ourselves very early to the easier case of euclidean signature. Technically, we shall use the tools of non-commutative geometry which were developed in the euclidean context. Understanding why the signature of the effective space-time is indefinite remains one of the principal open problems of quantum gravity and we shall have little to add here besides pointing out that the development of a Lorentzian non-commutative geometry seems to be, from this perspective, a natural task.

Let $M$ be a (pseudo-)Riemannian manifold with metric $\gamma=\gamma_{\mu \nu} d x^{\mu} d x^{\nu}$. One usually takes the length

$$
\int d s=\int\left[\gamma_{\mu \nu}(x) \frac{d x^{\mu}}{d \tau} \frac{d x^{\nu}}{d \tau}\right]^{1 / 2} d \tau
$$

of the trajectory $\tau \mapsto x(\tau) \in M$ as the action functional for the test particle (with unit mass). The extrema of such an action are arbitrarily parametrized geodesics. It will be more convenient to use

$$
S(x(\cdot))=\frac{1}{2} \int \gamma_{\mu \nu}(x) \frac{d x^{\mu}}{d \tau} \frac{d x^{\nu}}{d \tau} d \tau
$$

as the action, with the stationary points given by the geodesics parametrized by a constant times the length. If, following Polyakov's approach [44], we couple the latter action to the world-line metric $h(\tau) d \tau^{2}$, replacing it by

$$
S(x(\cdot), h(\cdot))=\frac{1}{2} \int \gamma_{\mu \nu}(x) \frac{d x^{\mu}}{d \tau} \frac{d x^{\nu}}{d \tau} h^{-1 / 2} d \tau+\frac{1}{2} \int h^{1 / 2} d \tau,
$$


then we can recover the length of the trajectory by minimizing $(1.3)$ over $h(\cdot)$ : classically, the two actions are equivalent. For $h(\tau) \equiv 1$, the action (1.3) reduces to (1.2) which describes a mechanical system called the "geodesic flow" on $M$. Setting $h(\tau)$ to 1 may be viewed as a gauge choice fixing the reparametrization invariance of the action (1.1).

It is easy to quantize the geodesic motion of a test particle on a Riemannian manifold ${ }^{2}$. The Hilbert space of states $\mathbb{H}$ may be taken as the space $L^{2}\left(M, d v_{\gamma}\right)$ of functions on $M$ square-integrable with respect to the Riemannian volume $d v_{\gamma}$. Physically, this is the space of functions on the configuration space of the particle. As the (positive) Hamiltonian $\mathcal{H}$ governing the quantum evolution, we may take $-\frac{1}{2} \Delta_{\gamma}$, where $\Delta_{\gamma}$ is the Laplace-Beltrami operator on $M$. In a first step towards understanding what quantum Riemannian geometry might be, one may try to reformulate standard Riemannian geometry using the fundamental notions of quantum mechanics: those of a Hilbert space of states and of an algebra of observables. Somewhat surprisingly, the exercise has proven to be rich in consequences. It has led A. Connes to the development of non-commutative geometry [13] providing an extension of geometry to situations very far from its original context. One such situation, which required an extension of Connes' geometry to an infinite-dimensional setup, was the analysis of models of two-dimensional massive quantum supersymmetric field theory [34][35]. Those models, although not exactly solvable, could be controlled by the analytic methods of constructive field theory [27]. We shall argue below that non-commutative geometry (already in its finite-dimensional version) may provide tools to study the deformed geometry of the exactly solvable (massless) models of CFT.

The abstract pair $\left(\mathbb{H},-\frac{1}{2} \Delta_{\gamma}\right)$ does not, in general, determine the geometry of $M$ [43]: one cannot hear the shape of the drum [36]. We need more structure. Such additional structure is provided by the algebra $\mathcal{A}$ of observables measuring the position of the test particle, realized as the algebra of multiplication operators by (say, smooth, bounded) functions on $M$. The manifold $M$ may be reconstructed from $\mathcal{A}$. The points of $M$ may be identified with the characters of (the norm closure of) $\mathcal{A}$. The differentiable structure of $M$ is then determined since we know the smooth functions on it. That the abstract triple $\left(\mathbb{H},-\frac{1}{2} \Delta_{\gamma}, \mathcal{A}\right)$ determines also the Riemannian metric is implied ${ }^{3}$ by a slight modification of A. Connes' argument [15] which goes as follows: the geodesic distance between points $x$ and $y$ of $M$ (which are multiplicative linear functionals on $\mathcal{A}$ ) is given by

$$
d_{\gamma}(x, y)=\sup |\mathrm{f}(\mathrm{x})-\mathrm{f}(\mathrm{y})|
$$

where the supremum is taken over smooth bounded functions $f$ s.t.

$$
\frac{1}{2}\left(\Delta_{\gamma} f^{2}+f^{2} \Delta_{\gamma}\right)-f \Delta_{\gamma} f
$$

which is the multiplication operator by $\langle d f, d f\rangle_{\gamma} \equiv \gamma^{\mu \nu}\left(\partial_{\mu} f\right)\left(\partial_{\nu} f\right)$, has norm $\leq 1$. Similarly, much of classical Riemannian geometry may be rewritten in terms of the quantum mechanics of a particle moving on $M$ in a way that uses little of the particular properties of $\left(\mathbb{H},-\frac{1}{2} \Delta_{\gamma}, A\right)$.

\footnotetext{
${ }^{2}$ on pseudo-Riemannian manifolds a fully consistent quantization requires, however, a multi-particle approach

${ }^{3}$ we thank J. Dereziński for this observation
} 
Connes' idea was then to use triples like $(\mathbb{H}, \mathcal{H}, A)$, where $\mathcal{H}$ is a self-adjoint operator on $\mathbb{H}$ and $\mathcal{A}$ a $*$-algebra of bounded operators on $\mathbb{H}$ (in general non-commutative) as the starting point for non-commutative geometry ${ }^{4}$.

Let us now proceed from mechanics to $1+1$-dimensional field theory, with the 1-dimensional space compactified to a circle. On the classical level, a large family of such theories is given by the so called "sigma models". They are obtained by considering fields $x(\sigma, \tau)$ with values in the Riemannian manifold $M$, with the action functional

$$
S(x(\cdot, \cdot))=\frac{1}{4 \pi} \int \gamma_{\mu \nu}(x)\left(\partial_{\tau} x^{\mu} \partial_{\tau} x^{\nu}-\partial_{\sigma} x^{\mu} \partial_{\sigma} x^{\nu}\right) d \sigma d \tau
$$

We assume periodicity of $x(\cdot, \cdot)$ in the space variable $\sigma$ with period $2 \pi$. The $\sigma$ integration in (1.6) is restricted to the interval $[0,2 \pi[$. It is sometimes useful to consider more general sigma models, with the action modified by the addition of the term

$$
S^{\prime}(x(\cdot, \cdot))=\frac{1}{2 \pi} \int \beta_{\mu \nu}(x) \partial_{\sigma} x^{\mu} \partial_{\tau} x^{\nu} d \sigma d \tau,
$$

where $\beta_{\mu \nu} d x^{\mu} \wedge d x^{\nu} \equiv \beta$ is a 2 -form on $M$. The classical solutions of the stationarity equations for the sigma model action are parametrized, at least for small times, by the Cauchy data $x(\cdot, 0)$ and $\partial_{\tau} x(\cdot, 0)$, so that the space $L M$ of (smooth) loops $\sigma \mapsto x(\sigma, 0)$ in $M$ plays the role of the configuration space of the model. $L M$ is, itself, a Riemannian manifold (in the Fréchet sense) with the metric induced from that of $M$ :

$$
\|\delta x\|^{2}=\frac{1}{2 \pi} \int \gamma_{\mu \nu}(x(\sigma)) \delta x^{\mu}(\sigma) \delta x^{\nu}(\sigma) d \sigma .
$$

The replacement of $M$ by $L M$ may seem to be the essential step of string geometry. Notice, however, that the sigma model action (1.6) differs from the one for the geodesic motion on $L M$ by a "potential" type term with spatial derivatives of $x^{\mu}$. Besides, it is not the classical $1+1-$ dimensional field theory but the corresponding quantum theory which describes the classical (and perturbative) level of string theory and, consequently, we should describe the geometry of the quantum sigma models, not that of the classical ones. The latter is fairly directly related to geometry of the loop space $L M$. The quantum sigma models, however, require a renormalization of the target space geometry. If one attempts to construct them in a perturbation expansion in powers of the Planck constant $\hbar$, each order introduces (infinite) counterterms modifying the initial Riemannian metric of $M$ [19]. As a result of the renormalization, the direct relation between quantum theory and geometry of the target manifold $M$ or of its loop space $L M$ is blurred. This is a new phenomenon since, as we have seen, quantum mechanics of a particle on a Riemannian manifold fully encodes the geometry of the manifold. To the first order in $\hbar$, there is no metric renormalization and a sigma model defines, in this approximation, a CFT if and only if the target metric is Ricci flat, i.e. if it solves the Einstein equations [19]. Clearly, we may view the Riemann-Einstein geometry as a limiting case of the stringy one.

\footnotetext{
${ }^{4}$ in fact, he uses the Dirac operator instead of the Laplacian, an important refinement which we shall discuss below
} 
Renormalization renders a rigorous construction of generic sigma models very difficult. There exists, however, a big pool of exactly solvable 1+1-dimensional quantum field theory models. Among those, there is a rich family of CFT's where we know exact expressions not only for the energy eigenvalues but also for Green's functions encoding the operator product of field operators. With any model of quantum field theory, we may associate a triple $(\mathbb{H}, \mathcal{H}, A)$, where $\mathbb{H}$ is the Hilbert space of states, $\mathcal{H}$ is the Hamiltonian and $\mathcal{A}$ is the (non-commutative) algebra generated by the field operators. We may think of this triple as encoding the effective quantum geometry of the space of field configurations or of its cotangent bundle. For the sigma models, this effective geometry summarizes the deformation, due to the renormalization effects, of the infinite-dimensional geometry of the loop space $L M$. The presentation of a quantum model by the triple $(\mathbb{H}, \mathcal{H}, A)$, selecting the Hamiltonian, is more natural in mechanics than in field theory, where it requires a choice of the time direction on the world-sheet. There, it would be more appropriate to specify the energy-momentum vector $(\mathcal{H}, \mathcal{P})$ or, in $\mathrm{CFT}$, the whole set of generators of the conformal algebra or of its extensions. In fact, the most convenient algebraic setup for describing non-commutative geometry of quantum fields still remains to be found. Below, we shall concentrate on geometry of the low energy modes of quantum field theory where quantum mechanical description is quite sufficient.

On the classical level, it is easy to recover the geodesic motion of the particle on $M$ from the sigma model: it is enough to consider 1+1-dimensional fields which do not depend on the space variable $\sigma$. Notice that, for such fields, the action (1.6) reduces to (1.2) and (1.7) disappears. The space of $\sigma$-independent fields realizes an embedding of $M$ into the subspace of elements of $L M$ invariant under reparametrizations. Although it is difficult to construct sigma models by quantizing the classical theory which has $M$ as the target, one may ask if it is possible to identify the effective target starting from a 1+1-dimensional quantum field model. Below, we shall show that, indeed, this can be done for the CFT models by using there infinite-dimensional symmetries like those given by the Virasoro algebra (essentially the algebra of infinitesimal reparametrizations of the circle), the current algebras or the supersymmetric extensions of those. With the use of such a symmetry algebra, one may associate to a CFT model a triple $\left(\mathbb{H}_{0}, \mathcal{H}_{0}, \mathcal{A}_{0}\right)$ describing the effective quantum geometry of zero modes of the string. The latter dominate the low energy regime where the internal motion of the string may be neglected. Usually the algebra $\mathcal{A}_{0}$ will be non-commutative, the commutativity being restored only in a semiclassical limit. Sometimes several distinct families of triples $\left(\mathbb{H}_{0}, \mathcal{H}_{0}, \mathcal{A}_{0}\right)$, yielding the effective low energy description in different limiting regimes, may be associated to a given family of conformal models. Consequently, one CFT may correspond to different (in general non commutative) effective targets. As we shall see, this is the essence of the duality [31] and mirror symmetry [30][9] phenomena which are among the most interesting novel features of string geometry in the large.

Certainly, string geometry is still in an early stage of development. What we have at our disposal are numerous examples of CFT models with rich infinite-dimensional symmetries. They may be thought of as symmetric spaces of string geometry. Their study is a stringy version of Klein's "Erlanger Programm". What is still largely missing is a stringy version of Riemann's approach to geometry. Such an approach should probably pass through string field 
theory which has common points with Connes' non-commutative geometry, as Witten's open string field theory [52] has shown. What is described below, is a more timid attempt to develop one limited aspect of string geometry: that of the effective metric geometry of low energy states. We point out that Connes' theory provides useful tools also for this more limited and more phenomenological approach. There are other geometric aspects of the low energy string like those involving dilatonic and Yang-Mills geometry. They require studying more general sigma models whose examples we shall encounter examining coset conformal theories and their supersymmetric versions. The non-metric aspects of string geometry should be related to the non-commutative $K$-theory and certainly deserve to be studied. One of the shortcomings of our approach (present already in the treatment of the relativistic quantum mechanics) is that we work with the world-sheet (world-line) metric fixed ignoring the effects of fluctuations of the latter. In this way, what we study is more geometry of the conformal fields than that of the string. This is not necessarily a drawback since CFT geometry may be more appropriate for the classical description of non-topological phases of the string. Nevertheless, a deeper understanding of string geometry should take into account the fluctuations of the world-sheet backgrounds which, treated with BRST techniques, play an important role in string field theory.

The following is the plan of the present exposé:

In Section 2, we shall describe the sigma models with a circle as the target. They are essentially versions of free 1+1-dimensional fields. These models illustrate the duality phenomenon responsible for the appearance of a fundamental length scale in string geometry. Their slight generalization with complex tori as the targets allows also to exhibit the simplest instance of the mirror symmetry [55].

Section 3 will be a guided tour through the factory of symmetric models of CFT. The basic raw material for the production of those models is the Wess-Zumino-Witten (WZW) $1+1$ dimensional field theory which, when processed by a gauging machine, gives a rich family of "coset models" of CFT. We shall briefly explain how the gauging machine works.

Section 4 will be devoted to geometry of the supersymmetric CFT models. We shall present the supersymmetric version of the WZW theory and of the coset models.

Finally, in Section 5, we shall sketch the relation between CFT models with $\mathrm{N}=2$ supersymmetry and Calabi-Yau geometry. This relation lies at the core of the mirror symmetry phenomenon for which non-commutative geometry provides a natural framework.

Acknowledgements. We thank A. Connes for supporting us in our struggle to learn some non-commutative geometry and $\mathrm{C}$. Voisin for teaching us basic complex geometry. In working on the program described in these notes we profited from numerous discussions with A. H. Chamseddine and G. Felder. J.F. thanks the I.H.E.S. and K.G. the Forschungsinstitut für Mathematik of E.T.H. for hospitality which made this collaboration possible. We are also greatful to J.Feldman, R. Froese and L. Rosen, the organizers of the Vancouver meeting, for the possibility to present our ideas to a mathematical physics audience. 


\section{Toroidal geometry}

\section{1. $S^{1}$ target and duality}

The simplest sigma model is obtained by taking the circle $\mathbb{R} / 2 \pi \mathbb{Z} \equiv S^{1}$ as the target. We shall use the angle variable $x$ as the coordinate of the circle. It may be assumed that the Riemannian metric of $S^{1}$ takes the form $\gamma=r^{2} d x^{2}$, since the radius $r$ is the only metric invariant. In other words, $r>0$ parametrizes the half line of Riemannian circles. Encoding the geometry of the circle in quantum mechanics of a particle moving on it gives rise to the triple

$$
\left(L^{2}\left(S^{1}, \frac{d x}{\sqrt{2 \pi}}\right),-\frac{1}{2} r^{-2} \frac{d^{2}}{d x^{2}}, C^{\infty}\left(S^{1}\right)\right)
$$

Functions $x \mapsto \mathrm{e}^{i n x}$ are the eigenfunctions of the Laplacian corresponding to the eigenvalues $r^{-2} n^{2}$. The action of a sigma model with the $S^{1}$ target is

$$
S(x(\cdot, \cdot))=\frac{r^{2}}{4 \pi} \int\left(\left(\partial_{\tau} x\right)^{2}-\left(\partial_{\sigma} x\right)^{2}\right) d \sigma d \tau .
$$

Its stationary points satisfy the wave equation

$$
\left(\partial_{\tau}^{2}-\partial_{\sigma}^{2}\right) x=0
$$

with the general solution

$$
x(\sigma, \tau)=x^{0}+r^{-2} p \tau+w \sigma+\sum_{n \neq 0} \frac{1}{\sqrt{2} i \tau n} \alpha_{n}^{+} \mathrm{e}^{i n(\sigma+\tau)}+\sum_{n \neq 0} \frac{1}{\sqrt{2} i \tau n} \alpha_{n}^{-} \mathrm{e}^{-i n(\sigma-\tau)} .
$$

$w \in \mathbb{Z}$ is the winding number, a homotopy invariant of the solution. It labels the connected components of the space of solutions. The canonical Poisson brackets

$$
\left\{x(\sigma, 0), \partial_{\tau} x\left(\sigma^{\prime}, 0\right)\right\}=2 \pi r^{-2} \delta\left(\sigma-\sigma^{\prime}\right)
$$

translate to

$$
\left\{x^{0}, p\right\}=1, \quad\left\{\alpha_{n}^{+}, \alpha_{m}^{+}\right\}=i n \delta_{n+m, 0}, \quad\left\{\alpha_{n}^{-}, \alpha_{m}^{-}\right\}=i n \delta_{n+m, 0}
$$

with all the other Poisson brackets vanishing. Classically, the model posseses the conformal symmetry acting by reparametrizations of $\tau \pm \sigma$ and the symmetry

$$
x(\sigma, \tau) \mapsto x(\sigma, \tau) \pm \delta^{ \pm} x(\tau \pm \sigma),
$$

where $\delta^{ \pm} x(\cdot)$ are arbitrary (periodic) functions.

Since we are essentially in the free field case, quantization of the model, replacing Poisson brackets by $i$ times commutators, is standard. The Hilbert space of states of the quantized system is

$$
\mathbb{H}=L^{2}\left(S^{1}, \frac{d x}{\sqrt{2 \pi}}\right)^{\mathbb{Z}} \otimes \mathcal{F}^{+} \otimes \mathcal{F}^{-}
$$


Above, the exponent $\mathbb{Z}$ indicates the infinite direct sum of $L^{2}$ spaces with the components labeled by the winding numbers $w \in \mathbb{Z}$. Periodic functions of $x^{0}$ act by multiplication and $p$ as $i \frac{d}{d x}$ in each $L^{2}$ component. We may $\operatorname{span} L^{2}\left(S^{1}, \frac{d x}{\sqrt{2 \pi}}\right)^{\mathbb{Z}}$ by the eigenvectors $\left|p_{w}, w\right\rangle=$ $\mathrm{e}^{-i p_{w} x}, p_{w} \in \mathbb{Z}$, of $i \frac{d}{d x}$ in each component of the direct sum. We shall also label states $\left|p_{w}, w\right\rangle$ as $\left|p^{+} ; p^{-}\right\rangle$, where the left-right momenta

$$
p^{ \pm}=\frac{1}{\sqrt{2}}\left(r^{-1} p_{w} \pm r w\right)
$$

The set $\left\{\left(p^{+}, p^{-}\right)\right\}$provided with the quadratic form $\left(p^{+}\right)^{2}-\left(p^{-}\right)^{2}=2 p_{w} w$ forms an even selfdual Lorentzian lattice. $\mathcal{F}^{ \pm}$are two copies of the bosonic Fock space built on the vacuum state $|0\rangle$ annihilated by $\alpha_{n}, n>0$, via the action of operators $\alpha_{n}, n<0 .\left[\alpha_{n}, \alpha_{m}\right]=n \delta_{n+m, 0}$ and $\alpha_{n}^{*}=\alpha_{-n}$. We shall consider $L^{2}\left(S^{1}\right)^{\mathbb{Z}}$ as embedded into $\mathbb{H}$ by $\left|p^{+} ; p^{-}\right\rangle \mapsto\left|p^{+} ; p^{-}\right\rangle \otimes|0\rangle_{+} \otimes|0\rangle_{-}$.

Let us introduce chiral (multivalued) quantum fields $X_{ \pm}$,

$$
X_{ \pm}(\tau \pm \sigma)=X_{ \pm}^{0}+p^{ \pm}(\tau \pm \sigma)+\sum_{n \neq 0} \frac{1}{i n} \alpha_{n}^{ \pm} \mathrm{e}^{i n(\tau \pm \sigma)}
$$

where $X_{ \pm}^{0}=\frac{1}{i} \frac{d}{d p^{ \pm}}$acts in the extended space with arbitrary momenta. On the quantum level, symmetry (2.7) is generated by a commuting pair of $u(1)$ current algebras with the currents

$$
\begin{gathered}
j^{ \pm}(\tau \pm \sigma)=\partial_{\sigma} X_{ \pm}(\tau \pm \sigma) \equiv \sum_{n=-\infty}^{\infty} j_{n}^{ \pm} \mathrm{e}^{i n(\tau \pm \sigma)}, \\
{\left[j_{n}, j_{m}\right]=n \delta_{n+m, 0}}
\end{gathered}
$$

$\left(j_{0}^{ \pm}= \pm p^{ \pm}, j_{n}^{ \pm}= \pm \alpha_{n}^{ \pm}\right.$for $\left.n \neq 0\right)$. The Hilbert space $\mathbb{H}$ is a direct sum of the irreducible (highest weight) representations of $j^{ \pm}$acting in $\left|p^{+} ; p^{-}\right\rangle \otimes \mathcal{F}^{+} \otimes \mathcal{F}^{-}$, labeled by $u(1)$ charges $\pm p^{ \pm}$. The classical conformal symmetry also carries over to the quantum level. It is represented there by a commuting pair of Virasoro algebras given by the Sugawara construction [47]:

$$
L_{n}^{ \pm}=\frac{1}{2} \sum_{m=-\infty}^{\infty}: j_{m}^{ \pm} j_{n-m}^{ \pm}:
$$

where the Wick ordering puts operators $\alpha_{n}^{ \pm}$with $n<0$ to the left of the ones with $n>0$.

$$
\left[L_{n}^{ \pm}, L_{m}^{ \pm}\right]=(n-m) L_{n+m}^{ \pm}+\frac{c}{12}\left(n^{3}-n\right) \delta_{n+m, 0},
$$

with the Virasoro central charge $c=1$. The Hamiltonian of the model is $\mathcal{H}=L_{0}^{+}+L_{0}^{-}-\frac{1}{12}$ and the momentum operator generating the space translations is $\mathcal{P}=L_{0}^{+}-L_{0}^{-}$.

$$
\mathcal{H}=\frac{1}{2}\left(r^{-2} p^{2}+r^{2} w^{2}\right)+\sum_{n>0} \alpha_{-n}^{+} \alpha_{n}^{+}+\sum_{n>0} \alpha_{-n}^{-} \alpha_{n}^{-}-\frac{1}{12}
$$

and $|\mathrm{vac}\rangle \equiv|0 ; 0\rangle$ is its unique eigenstate (with eigenvalue $-\frac{1}{12}$ ) at the bottom of the spectrum (the vacuum). 
The basic quantum fields of our model are given by ordered exponentials of fields $X_{ \pm}$

$$
\tilde{V}_{q^{+} q^{-}}\left(z_{ \pm}\right) \equiv: \mathrm{e}^{-i q^{+} X_{+}(\tau+\sigma)-i q^{-} X_{-}(\tau-\sigma)}:
$$

where $z_{ \pm} \equiv \mathrm{e}^{-i(\tau \pm \sigma)}$ and the Wick ordering puts also the operators $X_{ \pm}^{0}$ to the left of $p^{ \pm}$. When

$$
q^{ \pm}=\frac{1}{\sqrt{2}}\left(r^{-1} n \pm r m\right)
$$

for $n, m \in \mathbb{Z}$, then the above operators ${ }^{5} \operatorname{map}$ (a dense subset of) our Hilbert space into itself. Corrected by the cocycles $c_{q+q^{-}}\left(p^{+}, p^{-}\right) \equiv(-1)^{\left(q^{+}+q^{-}\right)\left(p^{+}-p^{-}\right) / 2}=(-1)^{n w}$ they give mutually local vertex operators

$$
V^{q^{+} q^{-}}\left(z_{ \pm}\right)=\tilde{V}^{q^{+} q^{-}}\left(z_{ \pm}\right) c_{q^{+} q^{-}}
$$

$V_{q^{+} q^{-}}$are primary fields of charges $\pm q^{ \pm}$for the $u(1)$ currents $j^{ \pm}$, i.e. they satisfy the following commutation relations:

$$
\left[j_{n}^{ \pm}, V_{q^{+} q^{-}}\left(z_{ \pm}\right)\right]= \pm q^{ \pm} z_{ \pm}^{n} V_{q^{+} q^{-}}\left(z_{ \pm}\right)
$$

which say that $V_{q^{+} q^{-}}$transform as local fields of $u(1)$ charges $\pm q^{ \pm}$under the local gauge transformations induced by $j^{ \pm}$. Fields $V_{q^{+} q^{-}}$are also primary fields of the Virasoro algebras:

$$
\left[L_{n}^{ \pm}, V_{q^{+} q^{-}}\left(z_{ \pm}\right)\right]=\Delta_{q^{ \pm}}^{ \pm}(n+1) z_{ \pm}^{n} V_{q^{+} q^{-}}\left(z_{ \pm}\right)+z_{ \pm}^{n+1} \partial_{z_{ \pm}} V_{q^{+} q^{-}}\left(z_{ \pm}\right)
$$

i.e. they transform under the conformal transformations as tensors with weights $\Delta_{q^{ \pm}}^{ \pm} \equiv \frac{1}{2}\left(q^{ \pm}\right)^{2}$. As usual in CFT, the primary fields may be labeled by specific vectors

$$
\lim _{z_{ \pm} \rightarrow 0} V_{q^{+} q^{-}}\left(z_{ \pm}\right)|\mathrm{vac}\rangle=\left|q^{+} ; q^{-}\right\rangle
$$

in the Hilbert space (above, the values of $z_{ \pm}$are extended to the complex domain by analytic continuation).

Defining the (non-commutative) algebra $\mathcal{A}$ to be generated by the vertex operators, we obtain a triple

$$
(\mathbb{H}, \mathcal{H}, \mathcal{A})_{r}
$$

associated to the sigma model with the circle of radius $r$ as the target. It may be viewed as encoding geometry of the sigma model.

The following simple observation has deep consequences. $\tilde{j}^{ \pm} \equiv \pm j^{ \pm}$is an equally good commuting pair of $u(1)$ currents in the sigma model with the $S^{\mathbf{1}}$ target as $j^{ \pm}$. Now, the representation content of the radius $r$ model with respect to $\tilde{j}^{ \pm}$is the same as of the radius $r^{-1}$ model with respect to $j^{ \pm}$. The Hilbert spaces of states for radii $r$ and $r^{-1}$ may then be

\footnotetext{
${ }^{5}$ or rather their smeared versions
} 
identified by the duality transformation sending $\left|p^{+}, p^{-}\right\rangle$to $(-1)^{p_{w} w}\left|p^{+},-p^{-}\right\rangle$(i.e., modulo signs, interchanging the roles of the momentum and of the winding number) and $\alpha_{n}^{ \pm}$to $\pm \alpha^{ \pm}$. Under this identification, the Hamiltonians and the algebras $\mathcal{A}$ (as well as the entire CFT's) coincide:

$$
(\mathbb{H}, \mathcal{H}, \mathcal{A})_{\tau} \cong(\mathbb{H}, \mathcal{H}, \mathcal{A})_{\tau^{-1}}
$$

This phenomenon distinguishes the stringy geometry of circles from their point-set Riemannian geometry. While the space of Riemannian circles was the half line parametrized by the radius $r$, that of the sigma models with circle targets is an orbifold of the latter obtained by the identification of $r$ with $r^{-1}$.

The above is an example of a more general duality phenomenon responsible for the appearance of a fundamental length scale in string theory. This is certainly one of the most promising features of the latter. In order to understand how such a length scale arises, let us think of how one probes the effective space(-time) geometry in string theory. As mentioned before, this should be done by looking at the low energy states ${ }^{6}$ of the string in which the stringy internal modes are not excited and one effectively sees quantum mechanics of point-like objects. Let us suppose that the string vacuum is described by a sigma model with the $S^{\mathbf{1}}$ target. The oscillatory modes of the string created by operators $\alpha_{n}^{ \pm}, n<0$ have energies quantized to integer values, so we should look at the states with energies $\ll 1$. If the radius $r$ of the circle is much bigger than 1 (or than the Planck length in dimensional units) then the low-energy spectrum of the sigma model is given by the states $\left|p_{0}, 0\right\rangle, p_{0} \in \mathbb{Z}$, describing the baricentric degree of freedom $x^{0}$. We effectively obtain the quantum mechanics of a particle moving on the circle of radius $r$. If $r$ is much smaller than 1 then the low-energy spectrum of the sigma model is given by the states $|0, w\rangle$ corresponding to the winding modes of the string. But this is exactly like the spectrum in the quantum mechanics of a particle moving on the circle of radius $r^{-1}$. As a result, we never see circles of small radii as effective geometries! Notice that this is a quantum phenomenon as is signaled by the presence of $\hbar$ in the expression for the Planck length.

We may formalize this observation in the following way. Notice that the classical states invariant under (2.7) with $\int \delta^{ \pm} x=0$ depend only on the zero mode variables $x_{0}, p$ and $w$. On the quantum level, we may then define the zero mode states as those annihilated by the positive frequency part of the $u(1)$ currents

$$
\left.\mathbb{H}_{0}=\left\{|\phi\rangle \in \mathbb{H}\left|j_{n}^{ \pm}\right| \phi\right\rangle=0 \text { for } n>0\right\} \text {. }
$$

$\mathbb{H}_{0}$ is the subspace of the highest weight vectors for the $u(1) \times u(1)$ current algebra. It is spanned by vectors $\left|p^{+} ; p^{-}\right\rangle$with $p^{ \pm}$as in (2.9). The Hamiltonian $\mathcal{H}$ leaves $\mathbb{H}_{0}$ invariant:

$$
\left(\mathcal{H}+\frac{1}{12}\right)\left|p^{+} ; p^{-}\right\rangle=\frac{1}{2}\left(r^{-2} p_{w}^{2}+r^{2} w^{2}\right)\left|p^{+} ; p^{-}\right\rangle \text {. }
$$

Notice that not all states in $\mathbb{H}_{0}$ have energy $\ll 1$. In the semiclassical regime when $r \gg 1$ this is possible only for states with winding number $w=0$. We shall then define a subspace

$$
\left.\mathbb{H}_{0}^{\prime}=\left\{|\phi\rangle \in \mathbb{H}_{0}\left|\left(j_{0}^{+}+j_{0}^{-}\right)\right| \phi\right\rangle=0\right\}
$$

\footnotetext{
${ }^{6}$ with the energy measured from the bottom of the spectrum
} 
This corresponds to taking only the highest weight vectors belonging to the complex conjugate pairs of left-right representations of the $u(1)$ current algebra. $\mathbb{H}_{0}^{\prime}$ is spanned by vectors $\left|p^{+} ; p^{-}\right\rangle$ with $p^{+}=p^{-}$. Let $E_{0}$ and $E_{0}^{\prime}$ denote the orthogonal projections of $\mathbb{H}$ on $\mathbb{H}_{0}$ and $\mathbb{H}_{0}^{\prime}$, respectively. Notice that

$$
E_{0} V_{q^{+} q^{-}}(1)\left|p^{+} ; p^{-}\right\rangle=(-1)^{\left(q^{+}+q^{-}\right)\left(p^{+}-p^{-}\right) / 2}\left|q^{+}+p^{+} ; q^{-}+p^{-}\right\rangle
$$

Let us consider the (commutative) algebra $\mathcal{A}_{0}^{\prime}$ generated by operators $\left.E_{0}^{\prime} V_{q q}(1)\right|_{\mathbb{I P}_{0}}$. We obtain this way the effective target geometry

$$
\left(\mathbb{H}_{0}^{\prime},\left.\left(\mathcal{H}+\frac{1}{12}\right)\right|_{\mathbb{H}_{0}}, \mathcal{A}_{0}^{\prime}\right)_{r}
$$

describing the low energy regime of the sigma model when $r \gg 1$. $\mathbb{H}_{0}^{\prime}$ is naturally isomorphic to $L^{2}\left(S^{1}, \frac{d x}{\sqrt{2 \pi}}\right)$ by identifying the vector $\left|p_{0}, 0\right\rangle \equiv\left|p_{0} ; p_{0}\right\rangle$ with the function $\mathrm{e}^{-i p_{0} x}$. With this identification,

$$
\left(\mathbb{H}_{0}^{\prime},\left.\left(\mathcal{H}+\frac{1}{12}\right)\right|_{\mathbb{H}_{0}^{\prime}}, \mathcal{A}_{0}^{\prime}\right)_{r} \cong\left(L^{2}\left(S^{1}, d x\right),-\frac{1}{2} r^{-2} \frac{d^{2}}{d x^{2}}, C^{\infty}\left(S^{1}\right)\right)
$$

so that the effective target (2.28) is just a circle of radius $r$. Notice that we have used only the pair of $u(1)$ current algebras $\left(j_{n}^{ \pm}\right)$in order to define the effective target geometry.

What is the meaning of duality in this language? Replacing the pair $j^{ \pm}$of currents by $\tilde{j}^{ \pm}= \pm j^{ \pm}$and repeating the whole construction of the target geometry, we obtain a different triple

$$
\left(\mathbb{H}_{0}^{\prime \prime},\left.\left(\mathcal{H}+\frac{1}{12}\right)\right|_{\mathbb{H}_{0}^{\prime \prime}}, \mathcal{A}_{0}^{\prime \prime}\right)_{r}
$$

where

$$
\left.\mathbb{H}_{0}^{\prime \prime}=\left\{|\phi\rangle \in \mathbb{H}_{0}\left|\left(j_{0}^{+}-j_{0}^{-}\right)\right| \phi\right\rangle=0\right\}
$$

and $\mathcal{A}_{0}^{\prime \prime}$ is generated by $\left.E_{0}^{\prime \prime} V_{q(-q)}(1)\right|_{\mathbb{H}_{0}^{\prime \prime}} . \mathbb{H}_{0}^{\prime \prime}$ is spanned by vectors $|0, w\rangle$ and is naturally isomorphic to $L^{2}\left(S^{1}, \frac{d x}{\sqrt{2 \pi}}\right)$ by identifying $|0, w\rangle$ with the function $\mathrm{e}^{-i w x}$. Now

$$
\left(\mathbb{H}_{0}^{\prime \prime},\left.\left(\mathcal{H}+\frac{1}{12}\right)\right|_{\mathbb{H}_{0}^{\prime \prime}}, \mathcal{A}_{0}^{\prime}\right)_{r} \cong\left(L^{2}\left(S^{1}, d x\right),-\frac{1}{2} r^{2} \frac{d^{2}}{d x^{2}}, C^{\infty}\left(S^{1}\right)\right)
$$

and the second effective target is the circle of radius $r^{-1}$. It describes the low energy regime of the sigma model when $r \ll 1$. As we see, duality results in the possibility to assign to the sigma model two different effective targets.

Above, we have described the procedure to define an effective target of a sigma model based on the $u(1)$ current algebra symmetry. On the classical level, we could try to select the constant modes of the string by imposing the reparametrization invariance. In the quantum theory, we could proceed using the Virasoro algebra. This would lead to the zero mode geometry $\left(\mathbb{H}_{0},\left.\mathcal{H}\right|_{\mathbb{H}_{0}}, \mathcal{A}_{0}\right)$, where now $\mathbb{H}_{0}$ is composed of states annihilated by $L_{n}^{ \pm}$for $n>0$ and by 
$L_{0}^{+}-L_{0}^{-}$, and $\mathcal{A}_{0}$ is generated by the Virasoro primary fields at $z_{ \pm}=1$ projected to $\mathbb{H}_{0}$. The resulting "small space" $\mathbb{H}_{0}$ is bigger than $\mathbb{H}_{0}^{\prime}$ and $\mathbb{H}_{0}^{\prime \prime}$ constructed above: $L_{n}^{ \pm}\left|p^{+}, p^{-}\right\rangle=0$ for $n>0$ and the condition $\left(L_{0}^{+}-L_{0}^{-}\right)\left|p^{+}, p^{-}\right\rangle=0$ requires that $p_{w}$ or $w$ be zero. Thus, the both dual current algebra targets end up in the Virasoro target. Note that the Virasoro-based construction of the zero mode geometry may be carried out in any CFT model.

\section{2. $T^{2}$ target and mirror symmetry}

It will be instructive to consider a slightly more involved example of a sigma model with a flat complex one-dimensional torus as the target. Let $\mathcal{T}^{2} \equiv S^{1} \times S^{1}$. The complex structure on $\mathcal{T}^{2}$ may be defined by the complex coordinate $z=x^{1}+T x^{2}$, where $x^{\mu}$ are the angle coordinates on $S^{1} \times S^{1}$ and $T \equiv T_{1}+i T_{2}$ is a complex number with imaginary part $T_{2}>0$. We shall also equip $\mathcal{T}^{2}$ with the flat Kähler metric $K=\frac{R_{2}}{T_{2}} d z d \bar{z}$, where $R_{2}>0$, and a closed 2 -form $\frac{i R_{1}}{T_{2}} d z \wedge d \bar{z}=\beta_{\mu \nu} d x^{\mu} \wedge d x^{\nu} \equiv \beta$, where $R_{1}$ is a real number. The Kähler metric induces a Riemannian one $\gamma_{\mu \nu} d x^{\mu} d x^{\nu} \equiv \gamma$.

$$
\left(\gamma_{\mu \nu}\right)=\frac{R_{2}}{T_{2}}\left(\begin{array}{cc}
1 & T_{1} \\
T_{1} & |T|^{2}
\end{array}\right), \quad\left(\beta_{\mu \nu}\right)=\left(\begin{array}{cc}
0 & R_{1} \\
-R_{1} & 0
\end{array}\right)
$$

For later convenience, we shall combine $R_{1}$ and $R_{2}$ into the complex number $R=R_{1}+i R_{2}$ and shall introduce matrices $\left(d_{\mu \nu}^{ \pm}\right) \equiv\left(\gamma_{\mu \nu} \pm \beta_{\mu \nu}\right)$. Notice that, if $\omega=\frac{R_{2}}{T_{2}} d z \wedge d \bar{z}$ denotes the Kähler form, then $\int_{\mathcal{T}^{2}}(\beta+\omega)=2 R$. As the action of the sigma model, we shall take the sum of (1.6) and (1.7) with $\gamma_{\mu \nu}$ and $\beta_{\mu \nu}$ as above.

The model with the $\mathcal{T}^{2}$ target may be treated in full analogy to the one with the $S^{1}$ target. Let us just collect the relevant formulae:

The classical solutions:

$$
\begin{aligned}
x^{\mu}(\sigma, \tau) & =x^{\mu 0}+\gamma^{\mu \nu}\left(p_{\nu}+\beta_{\nu \lambda} w^{\lambda}\right) \tau+w^{\mu} \sigma \\
& +\sum_{n \neq 0} \frac{1}{\sqrt{2} i n} \alpha_{n}^{\mu+} \mathrm{e}^{i n(\sigma+\tau)}+\sum_{n \neq 0} \frac{1}{\sqrt{2} i n} \alpha_{n}^{\mu-} \mathrm{e}^{-i n(\sigma-\tau)} .
\end{aligned}
$$

The Poisson brackets:

$$
\left\{x^{\mu 0}, p_{\nu}\right\}=\delta_{\nu}^{\mu}, \quad\left\{\alpha_{n}^{\mu+}, \alpha_{m}^{\nu+}\right\}=i n \gamma^{\mu \nu} \delta_{n+m, 0}, \quad\left\{\alpha_{n}^{\mu-}, \alpha_{m}^{\nu-}\right\}=i n \gamma^{\mu \nu} \delta_{n+m, 0} .
$$

The Hilbert space of states:

$$
\mathbb{H}=L^{2}\left(S^{1} \times S^{1}, \frac{d x^{1} d x^{2}}{2 \pi}\right)^{\mathbb{Z}} \otimes \mathcal{F}^{+} \otimes \mathcal{F}^{-}
$$

with $p_{\mu}$ acting as $i \frac{d}{d x^{\mu}}$ and the Fock space operators $\alpha_{n}^{\mu \pm}$ satisfying $\left[\alpha_{n}^{\mu \pm}, \alpha_{m}^{\nu \pm}\right]=n \gamma^{\mu \nu} \delta_{n+m, 0}$, $\left(\alpha_{n}^{\mu \pm}\right)^{*}=\alpha_{-n}^{\mu \pm}$.

The chiral fields:

$$
X_{ \pm}^{\mu}(\tau \pm \sigma)=X_{ \pm}^{\mu 0}+\gamma^{\mu \nu} p_{\nu}^{ \pm}(\tau \pm \sigma)+\sum_{n \neq 0} \frac{1}{i n} \alpha_{n}^{\mu \pm} \mathrm{e}^{i n(\tau \pm \sigma)}
$$


where $X_{ \pm}^{\mu 0}=\frac{1}{i} \frac{d}{d p_{\mu}^{ \pm}}$and the left-right momenta

$$
p_{\nu}^{ \pm}=\frac{1}{\sqrt{2}}\left(p_{\nu} \pm d_{\nu \lambda}^{ \pm} w^{\lambda}\right) .
$$

Note that the set of momenta $\left\{\left(p^{+}, p^{-}\right)\right\}$, together with the quadratic form $p_{\mu}^{+} \gamma^{\mu \nu} p_{\nu}^{+}-$ $p_{\mu}^{-} \gamma^{\mu \nu} p_{\nu}^{-}=2 p_{\mu} w^{\mu}$ forms an even self-dual Lorentzian lattice.

The vertex operators:

$$
V_{q^{+} q^{-}}\left(z_{ \pm}\right)=: \mathrm{e}^{-i q_{\mu}^{+} X_{+}^{\mu}(\tau+\sigma)-i q_{\mu}^{-} X_{-}^{\mu}(\tau-\sigma)}: c_{q^{+} q^{-}},
$$

where $q_{\mu}^{ \pm}=\frac{1}{\sqrt{2}}\left(n_{\mu} \pm d_{\mu \nu}^{ \pm} m^{\nu}\right)$ with $n_{\mu}, m^{\nu} \in \mathbb{Z}$ and the cocycles

$$
c_{q^{+} q^{-}}\left(p^{+}, p^{-}\right)=(-1)^{n_{\mu} w^{\mu}} .
$$

$V_{q^{+} q^{+}}$are mutually local fields and their smeared versions generate a non-commutative algebra $\mathcal{A}$.

The current and Virasoro algebras:

$$
\begin{gathered}
j_{ \pm}^{\mu}(\tau \pm \sigma)=\partial_{\sigma} X_{ \pm}^{\mu}(\tau \pm \sigma) \equiv \sum_{n=-\infty}^{\infty} j_{n}^{\mu \pm} \mathrm{e}^{i n(\tau \pm \sigma)} \\
L_{n}^{ \pm}=\frac{1}{2} \sum_{m=-\infty}^{\infty} \gamma_{\mu \nu}: j_{m}^{\mu \pm} j_{n-m}^{\nu \pm}:
\end{gathered}
$$

The Hamiltonian:

$$
\mathcal{H}=\frac{1}{2} \gamma^{\mu \nu} p_{\mu}^{+} p_{\nu}^{+}+\frac{1}{2} \gamma^{\mu \nu} p_{\mu}^{-} p_{\nu}^{-}+\sum_{n>0} \gamma_{\mu \nu} \alpha_{-n}^{\mu+} \alpha_{n}^{\nu+}+\sum_{n>0} \gamma_{\mu \nu} \alpha_{-n}^{\mu-} \alpha_{n}^{\nu-}-\frac{1}{6}
$$

All that was essentially a repetition of the story for the $S^{1}$ target. As the result of the above constructions, one obtains a triple

$$
(\mathbb{H}, \mathcal{H}, \mathcal{A})_{T, R} .
$$

What are the symmetries of $(\mathbb{H}, \mathcal{H}, \mathcal{A})_{T, R}$ or, more generally, of the conformal sigma models parametrized by $(T, R)$ ?

(i) The duality transformation relates the Hilbert spaces of the $(T, R)$ and $\left(-T^{-1},-R^{-1}\right)$ models corresponding to mutually inverse matrices $d^{ \pm}$. It interchanges the momenta and the winding numbers mapping $\left|p^{+} ; p^{-}\right\rangle$to $(-1)^{p_{\mu} w^{\mu}}\left|\left(d^{+}\right)^{-1} p^{+} ;-\left(d^{-}\right)^{-1} p^{-}\right\rangle$and it transforms $\alpha_{n}^{\mu \pm}$ to $\pm\left(d^{\mp}\right)^{-1}{ }_{\mu \nu} \alpha_{n}^{\nu \pm}$. It results in the equivalence

$$
(\mathbb{H}, \mathcal{H}, \mathcal{A})_{T, R} \cong(\mathbb{H}, \mathcal{H}, \mathcal{A})_{-T^{-1},-R^{-1}}
$$

(ii) Further symmetries are due to the fact that complex tori with $T$ replaced by $T+1$ or $-T^{-1}$ are conformally equivalent to the original ones (with the same value of $R$ ):

$$
(\mathbb{H}, \mathcal{H}, \mathcal{A})_{T, R} \cong(\mathbb{H}, \mathcal{H}, \mathcal{A})_{T+1, R} \cong(\mathbb{H}, \mathcal{H}, \mathcal{A})_{-T^{-1}, R}
$$


(iii) The change $R \mapsto R+1$ may be absorbed in a shift of the momenta $p_{\nu} \mapsto p_{\nu}-\epsilon_{\nu \lambda} w^{\lambda}$ so that

$$
(\mathbb{H}, \mathcal{H}, \mathcal{A})_{T, R} \cong(\mathbb{H}, \mathcal{H}, \mathcal{A})_{T, R+1}
$$

The above symmetries imply that

$$
(\mathbb{H}, \mathcal{H}, \mathcal{A})_{T, R} \cong(\mathbb{H}, \mathcal{H}, \mathcal{A})_{T^{\prime}, R^{\prime}}
$$

where

$$
T^{\prime}=\frac{a T+b}{c T+d}, \quad R^{\prime}=\frac{a^{\prime} R+b^{\prime}}{c^{\prime} R+d^{\prime}}
$$

for $\left(\begin{array}{ll}a & b \\ c & d\end{array}\right),\left(\begin{array}{ll}a^{\prime} & b^{\prime} \\ c^{\prime} & d^{\prime}\end{array}\right) \in S L(2, \mathbb{Z})$.

(iv) Explicit calculation shows that

$$
\begin{aligned}
\frac{1}{2} \gamma^{\mu \nu} p_{\mu}^{+} p_{\nu}^{+}+\frac{1}{2} \gamma^{\mu \nu} p_{\mu}^{-} p_{\nu}^{-} & =\frac{1}{4} \frac{1}{T_{2} R_{2}}\left|T p_{1}-p_{2}+R w_{1}+T R w_{2}\right|^{2} \\
& +\frac{1}{4} \frac{1}{T_{2} R_{2}}\left|T p_{1}-p_{2}+\bar{R} w_{1}+T \bar{R} w_{2}\right|^{2}
\end{aligned}
$$

It is easy to verify that the transformation relating the spaces of states for $(T, R)$ and $(R, T)$ by interchanging $p_{1}$ and $w_{1}$ in the vectors $\left|p^{+} ; p^{-}\right\rangle$(accompanied by multiplication by $(-1)^{p_{1} w^{1}}$ ) and mapping $\alpha_{n}^{1 \pm}$ to $\pm T_{2} R_{2}^{-1} \alpha_{n}^{1 \pm}-\left(T_{1} R_{2} \mp T_{2} R_{1}\right) R_{2}^{-1} \alpha_{n}^{2 \pm}$ establishes the equivalence

$$
(\mathbb{H}, \mathcal{H}, \mathcal{A})_{T, R} \cong(\mathbb{H}, \mathcal{H}, \mathcal{A})_{R, T}
$$

This is the simplest instance of the "mirror symmetry" [30][55] which claims the equivalence of conformal field theories for complex targets with roles of the complex and the Kähler structures interchanged. We shall return to this topic in Sect. 5.2.

Notice that the space of complex 2-dimenstional tori with flat Kähler metric and covariantly constant closed 2-forms is $\left(H_{+} \times H_{+}\right) / S L(2, \mathbb{Z})$, where $H_{+}$is the upper half-plane of $T$ and $R$ and $S L(2, \mathbb{Z})$ acts on $H_{+} \times H_{+}$by $(T, R) \mapsto\left(\frac{a T+b}{c T+d}, R\right)$. In contrast, the space of the sigma models with such targets is $\left(H_{+} \times H_{+}\right) /(S L(2, \mathbb{Z}) \times S L(2, \mathbb{Z})) / \mathbb{Z}_{2}$, where $\mathbb{Z}_{2}$ acts by interchanging $T$ and $R$.

Using the $u(1)$ currents $j^{\mu \pm}$ we could again define the zero-mode restriction of the theory by imposing the conditions $j_{n}^{\mu \pm}|\phi\rangle=0$. Different effective toroidal target geometries with different Riemannian metrics may then be obtained by restricting, additionally, the zero modes. It will be convenient to introduce the following combinations of currents:

$$
\tilde{j}_{n}^{\mu \pm}= \pm d_{\mu \nu}^{\mp} j_{n}^{\nu \pm}, \quad \mathbf{j}_{n}^{ \pm} \equiv \frac{i}{T_{2}}\left(j_{n}^{1 \pm}+T j_{n}^{2 \pm}\right), \quad \overline{\mathbf{j}}_{n}^{ \pm} \equiv-\frac{i}{T_{2}}\left(j_{n}^{1 \pm}+\bar{T} j_{n}^{2 \pm}\right)
$$

Then the zero mode conditions:

1. $\left(j_{0}^{\mu+}+j_{0}^{\mu-}\right)|\phi\rangle=0$ give the metric $\gamma_{\mu \nu} d x^{\mu} d x^{\nu}$ on the $\mathcal{T}^{2}$ target, 
2. $\left(\tilde{j}_{0}^{\mu+}+\widetilde{j}_{0}^{\mu-}\right)|\phi\rangle=0$ give the dual metric $\widetilde{\gamma}_{\mu \nu} d x^{\mu} d x^{\nu}$, where $\tilde{\gamma}_{\mu \nu} \equiv\left(d^{+}\right)^{-1^{\mu \lambda}} \gamma_{\lambda \rho}\left(d^{-}\right)^{-1 \rho \nu}$,

3. $\left(\mathbf{j}_{0}^{ \pm}+\overline{\mathbf{j}}_{0}^{\mp}\right)|\phi\rangle=0 \quad$ give the mirror metric $\tilde{\widetilde{\gamma}}_{\mu \nu} d x^{\mu} d x^{\nu}$ with $\left(\tilde{\tilde{\gamma}}_{\mu \nu}\right)$ as in (2.33), but with $T$ and $R$ interchanged.

The quantum mechanics of a particle on the torus with the above metrics describes then the low energy spectrum of the sigma model in the regimes $\gamma_{\mu \nu} \gg 1, \tilde{\gamma}_{\mu \nu} \gg 1$ and $\tilde{\tilde{\gamma}}_{\mu \nu} \gg 1$, respectively. Notice, that the passage from the first target to the dual one is obtain by the interchange of $j^{\mu \pm}$ and $\widetilde{j}^{\mu \pm}$ and to the mirror one by the interchange of $j^{-}$and $\bar{j}^{-}$.

The discussion of the last two sections may be generalized to the case of general toroidal sigma models leading to more general even self-dual Lorentzian lattices of left-right momenta. An interesting exercise, which remains to be done, is the calculation of the effective targets for the sigma models with fields taking values in toroidal orbifolds.

\section{WZW and coset theories}

\subsection{Geodesic motion on a group}

The geodesic motion on $S^{1}$ or $S^{1} \times S^{1}$ was essentially free and, consequently, easily solvable both classically and in quantum mechanics. $S^{1}$ is the simplest compact Lie group. For other compact Lie groups $G$, the geodesic motion w.r.t. the invariant metric, although not free, may be also easily solved using its symmetries. The relevant action functional of the particle trajectory $\tau \mapsto g(\tau) \in G$ is

$$
S(g(\cdot))=-\frac{k}{4} \int \operatorname{tr}\left(g^{-1} \partial_{\tau} g\right)^{2} d \tau,
$$

where the coupling constant $k>0$. The classical equations

$$
\partial_{\tau}\left(g \partial_{r} g^{-1}\right)=0
$$

give the geodesics on $G$. The quantized system has $L^{2}(G, d g)$ as the space of states, where $d g$ stands for the normalized Haar measure. It carries two (left-right) commuting (regular) unitary representations of $G$ acting by

$$
L_{g_{1}} R_{g_{2}} f(g)=f\left(g_{1}^{-1} g g_{2}\right) .
$$

The decomposition of the regular representation into the irreducible components results in the isomorphism

$$
L^{2}(G, d g) \cong \bigoplus_{R} V_{R} \otimes V_{\bar{R}}
$$

where $R$ runs through all unitary irreducible representations of $G$ in (finite-dimensional) Hilbert spaces $V_{R}$ and $\bar{R}$ denotes the representation complex conjugate to $R . V_{R} \otimes V_{\bar{R}}$ is 
spanned by matrix elements of the representation $\bar{R}$. The left (right) representation of $G$ acts on the left (right) factor. For $G=S U(2)$, we shall label representations $R$ by spins $j=0, \frac{1}{2}, 1, \ldots$

Let $\left(t^{A}\right)$ be a basis of the Lie algebra $\mathrm{g}$ of $G$ s.t. $\operatorname{tr} t^{A} t^{B}=\frac{1}{2} \delta^{A B}$. Let $J^{A+}=\frac{1}{i} d L\left(t^{A}\right)$ and $J^{A-}=\frac{1}{i} d R\left(t^{A}\right)$ be the (selfadjoint) operators expressing the infinitesimal actions of $t^{A}$ in $L^{2}(G)$. The quantum Hamiltonian of the model is given by

$$
\mathcal{H}=\frac{2}{k} J^{A+} J^{A+}=\frac{2}{k} J^{A-} J^{A-} \equiv-\frac{2}{k} \Delta_{G}
$$

i.e. it is proportional to the Laplacian on $G$ which acts as multiplication by minus the quadratic Casimir $c_{R}=c_{\bar{R}}$ in the $V_{R} \otimes V_{\bar{R}}$ subspace of (3.4). The triple

$$
\left(L^{2}(G, d g),-\frac{2}{k} \dot{\Delta}_{G}, C^{\infty}(G)\right)
$$

encodes the invariant Riemannian geometry on $G$.

\subsection{WZW model}

What about a sigma model with the group $G$ target? If we take

$$
S(g(\cdot, \cdot))=-\frac{k}{8 \pi} \int \operatorname{tr}\left(\left(g^{-1} \partial_{\tau} g\right)^{2}-\left(g^{-1} \partial_{\sigma} g\right)^{2}\right) d \sigma d \tau
$$

as the action functional then the model requires an infinite renormalization of the coupling constant $k$ and is believed to result in a massive two-dimensional field theory. One may, however, add to the action the term (1.7) with a 2 -form $\beta \equiv \beta_{\mu \nu} d x^{\mu} \wedge d x^{\nu}$ satisfying

$$
d \beta=\frac{k}{3} \operatorname{tr}\left(g^{-1} d g\right)^{\wedge 3} .
$$

Such 2 -forms on $G$ exist only locally. The freedom of their choice results in the $2 \pi k \mathbb{Z}$-valued ambiguity in the definition of the action which is irrelevant at the classical level but restricts the values of the coupling constant $k$ to (positive) integers in the quantum theory. For such values, the modified sigma model with the group target does not require renormalization of $k$ and gives rise to the WZW model of CFT (of "level" $k$ ) [51].

The Hilbert space $\mathbb{H}$ of the WZW model is built from the representations of two commuting copies of the Kac-Moody algebra $\hat{\mathrm{g}}$ generated by elements $J_{n}^{A \pm}$ satisfying the commutation relations

$$
\left[J_{n}^{A \pm}, J_{m}^{B \pm}\right]=i f^{A B C} J_{n+m}^{C \pm}+\frac{1}{2} k n \delta^{A B} \delta_{n+m, 0}
$$

where $f^{A B C}$ are the structure constants: $\left[t^{A}, t^{B}\right]=i f^{A B C} t^{C} . \widehat{\mathbf{g}}$ is the central extension of the loop algebra $L \mathrm{~g}$ and $k$ is its central charge (level). The unitary $\left(\left(J_{n}^{A}\right)^{*}=J_{-n}^{A}\right)$ irreducible highest weight representations of $\hat{\mathbf{g}}$ are labeled by $k$ (a non-negative integer) and an irreducible representation $R$ of $G$ from a restricted class (for $G=S U(2)$, with spin $j \leq \frac{k}{2}$ ) whose 
elements we shall call integrable (at level $k$ ) [37]. They act in infinite-dimensional (if $k>0$ ) spaces $\mathbf{V}_{R}^{k}$. For $G$ connected and simply connected

$$
\mathbb{H}=\left\{\bigoplus_{\substack{R \\ \text { integrable }}} \mathbf{V}_{R}^{k} \otimes \mathbf{V}_{R}^{k}\right\}^{-},
$$

where $\{\cdots\}^{-}$denotes the Hilbert space completion. The current modes $J_{n}^{A \pm}$ 's generate, by the Sugawara construction, two commuting representations of the Virasoro algebra

$$
L_{n}^{ \pm}=\frac{1}{k+g^{\vee}} \sum_{m=-\infty}^{\infty}: J_{m}^{A \pm} J_{n-m}^{A \pm}:
$$

with central charge $c_{k}^{G}=\frac{k \operatorname{dim}(G)}{k+g^{\vee}}\left(g^{\vee}\right.$ denotes the quadratic Casimir of the adjoint representation). The Hamiltonian of the model is

$$
\mathcal{H}=L_{0}^{+}+L_{0}^{-}-\frac{1}{12} c_{k}^{G},
$$

whereas

$$
\mathcal{P}=L_{0}^{+}-L_{0}^{-}
$$

generates the space translations.

Let us define a small space of states $\mathbb{H}_{0}$ as composed of vectors $|\phi\rangle \in \mathbb{H}$ satisfying the highest weight condition $J_{n}^{A \pm}|\phi\rangle=0$ for $n>0$. $\mathbb{H}_{0}$ carries the representation of $\mathbf{g} \oplus \mathbf{g}$ (and of $G \times G)$ given by the action of $J_{0}^{A \pm}$ 's. With respect to this representation,

$$
\mathbb{H}_{0} \cong \bigoplus_{\substack{R \\ \text { integrable }}} V_{R} \otimes V_{\bar{R}}
$$

which may be naturally identified (see (3.4)) with a subspace of $L^{2}(G)$ which we shall denote $L_{k}^{2}(G)$. The Hamiltonian $\mathcal{H}$ preserves $\mathbb{H}_{0}$ and reduces there to $-\frac{2}{k+g^{\vee}} \Delta_{G}-\frac{1}{12} c_{k}^{G}$. To each vector $|\phi\rangle \in \mathbb{H}_{0}$ there corresponds a unique primary field $V_{|\phi\rangle}\left(z_{ \pm}\right)$from a local family such that

$$
\left[J_{n}^{A \pm}, V_{|\phi\rangle}\left(z_{ \pm}\right)\right]=z_{ \pm}^{n} V_{J_{0}^{A \pm}|\phi\rangle}\left(z_{ \pm}\right)
$$

and

$$
\lim _{z_{ \pm} \rightarrow 0} V_{|\phi\rangle}\left(z_{ \pm}\right)|\mathrm{vac}\rangle=|\phi\rangle
$$

$V_{|\phi\rangle}\left(z_{ \pm}\right)$is also a primary field of the Sugawara Virasoro algebras (see (2.20)). Taking the algebra $\mathcal{A}$ generated by the primary fields, we obtain a triple $(\mathbb{H}, \mathcal{H}, \mathcal{A})$ encoding the geometry of the WZW CFT.

We have presented the WZW theory as a sigma model with a group target, but its construction on the quantum level has proceeded directly through the representation theory of the 
Kac-Moody algebra. Again the question arises how to see the target manifold geometry in the resulting quantum theory. It seems reasonable to proceed via the restriction to the "zero mode" subspace $\mathbb{H}_{0}$ of primary states with the Hamiltonian $\mathcal{H}_{0}=\left.\left(\mathcal{H}+\frac{1}{12} c_{k}^{G}\right)\right|_{\mathbb{H}_{0}}$. Such a restriction cuts out the descendent states created by operators $J_{n}^{A \pm}$, with $n<0$, which increase the energy by $|n|$. Since, for $G$ simple and simply connected, the left-right representations of the current algebra $\widehat{\mathbf{g}}$ appear only in complex conjugate pairs (unlike for the $S^{1}$ sigma model), we shall not impose any further zero mode conditions on the small space of states. In fact, $\mathbb{H}_{0}$ contains all states with low energy $(\ll 1)$ for $k \gg 1$. To encode the effective target geometry, we still need an algebra $\mathcal{A}_{0}$ of "functions" on the target. Let $E_{0}$ denote the orthogonal projection of $\mathbb{H}$ onto $\mathbb{H}_{0}$. We shall take as $\mathcal{A}_{0}$ the algebra generated by operators $\left.E_{0} V_{|\phi\rangle}(1)\right|_{\mathbb{H}_{0}}$. This way we obtain a triple

$$
\left(\mathbb{H}_{0}, \mathcal{H}_{0}, \mathcal{A}_{0}\right)
$$

What is its relation to the triple (3.6) representing the Riemannian geometry of the group $G$ ? As we have already noticed, $\mathbb{H}_{0}$ is a subspace of $L^{2}(G)$ and $\mathcal{H}_{0}$ coincides on it with $-\frac{2}{k+g^{v}} \Delta_{G}$. Thus we only need to understand the relation of $\mathcal{A}_{0}$ to the algebra of functions on $G$.

Let $f_{l}, l=1,2,3$, be three functions on $G$ lying, in the decomposition (3.4), inside $V_{R_{l}} \otimes V_{\bar{R}_{l}}$, respectively, where $R_{l}$ are representations integrable at level $k . f_{l}$ define vectors $\left|f_{l}\right\rangle \in \mathbb{H}_{0}$. Let $\operatorname{Inv}_{\left(R_{l}\right)}$ denote the subspace of $\otimes_{l} V_{R_{l}}$ invariant with respect to the diagonal action of $G$. The matrix elements of the primary fields $V_{|\phi\rangle}(1)$ between the states in $\mathbb{H}_{0}$ have the following form

$$
\left\langle\overline{f_{3}}\left|V_{\left.\mid f_{2}\right)}(1)\right| f_{1}\right\rangle=\left\langle C_{\left(R_{l}\right)}^{k} \mid \otimes_{l} f_{l}\right\rangle
$$

where $C_{\left(R_{l}\right)}^{k} \in \operatorname{Inv}_{\left(R_{l}\right)} \otimes \operatorname{Inv}_{\left(\bar{R}_{l}\right)} \cong \operatorname{End}\left(\operatorname{Inv}_{\left(R_{l}\right)}\right)$ is a positive element. For $G=S U(2)$, $\operatorname{Inv}_{\left(j_{l}\right)} \otimes \operatorname{Inv}_{\left(\bar{j}_{l}\right)}$, if non-vanishing, is canonically isomorphic to $\mathbb{C}$ and formula (3.18) takes the simpler form

$$
\left\langle\overline{f_{3}}\left|V_{\left|f_{2}\right\rangle}(1)\right| f_{1}\right\rangle=C_{\left(j_{1}, j_{2}, j_{3}\right)}^{k} \int_{G} \prod_{l} f_{l}(g) d g
$$

where $C_{\left(j_{l}\right)}^{k}$ are (up to normalization) the operator product coefficients [4] for the WZW theory. They have been computed in [58]:

$$
C_{\left(j_{1}, j_{2}, j_{3}\right)}^{k}=(J+1) ! P(J+1) P(1)^{1 / 2} \prod_{l=1}^{3} \frac{P\left(\hat{j}_{l}\right) \hat{j}_{l} !}{\left(2 j_{l}+1\right)^{1 / 2}\left(2 j_{l}\right) ! P\left(2 j_{l}\right)^{1 / 2} P\left(2 j_{l}+1\right)^{1 / 2}},
$$

where $J \equiv j_{1}+j_{2}+j_{3}, \bar{j}_{l} \equiv J-2 j_{l}$ and

$$
P(0)=1, \quad P(j)=\prod_{i=1}^{j} \frac{\Gamma\left(\frac{i}{k+2}\right)}{\Gamma\left(1-\frac{i}{k+2}\right)} .
$$

In (3.20), it is assumed that $j_{l} \leq \frac{k}{2}$. 
As we see from the relation (3.19), for $G=S U(2), \mathcal{A}_{0}$ is a deformed version of the algebra of multiplication by functions from $\mathbb{H}_{0}$. It is, however, non-commutative (in general). The commutativity is restored in the classical limit $k \rightarrow \infty$, where $\mathbb{H}_{0} \rightarrow L^{2}(G)$ and $C_{\left(j_{1}, j_{2}, j_{3}\right)}^{k} \rightarrow 1$. Also for general $G$,

$$
\lim _{k \rightarrow \infty} C_{\left(R_{l}\right)}^{k}=\mathrm{I} \in \operatorname{End}\left(\operatorname{Inv}_{\left(R_{l}\right)}\right)
$$

so that

$$
\lim _{k \rightarrow \infty}\left\langle C_{\left(R_{l}\right)}^{k} \mid \otimes_{l} f_{l}\right\rangle=\int_{G} \prod_{l} f_{l}(g) d g
$$

and one recovers, in the $k \rightarrow \infty$ limit, the triple (3.6) encoding the commutative geometry of the group manifold. For finite $k,\left(\mathbb{H}_{0}, \mathcal{H}_{0}, \mathcal{A}_{0}\right)$ represents a finite $\left(\mathbb{H}_{0}\right.$ is finite-dimensional) non-commutative geometry of the effective target of the WZW model.

We should stress once more that the infinite symmetry of the conformal model is an important input in our definition of the effective target. If, for example, in the case of the WZW model with $G=S U(2)$ we have used only the $u(1) \times u(1)$ current algebra, we should have obtained a different effective target. In particular, the $u(1)$ target would coincide for the level $k=1$ with that of the sigma model with field values in $S^{1}$ of radius $r=1$.

\subsection{Coset quantum mechanics}

There is a simple way, called the coset construction [28], to generate new CFT's from the group $G$-valued WZW model. Let us start by describing the quantum mechanical counterpart of the coset construction. As we have seen, $L^{2}(G)$ carries a unitary representation of $G \times G$. For any subgroup $H \subset G \times G$, we may consider the subspace $L^{2}(G)_{H} \subset L^{2}(G)$ of functions invariant under $H$. If $H$ is a subgroup of $G_{\text {left }}$ or a subgroup of $G_{\text {right }}$ or a product of such subgroups, we end up with the space of square-integrable functions on the (left, right or leftright) coset space. There is another possibility which has attracted less attention by harmonic analysts. One may take $H \subset G_{\text {diag }} \subset G \times G$. This leads to functions on $G / \operatorname{Ad}(H)$. Let us decompose the irreducible representations of $G$ with respect to $H$ :

$$
V_{R} \cong \bigoplus_{r} M_{r}^{R} \otimes V_{r}
$$

where $R$ refers to irreducible representations of $G$ and $r$ to those of $H$. The multiplicity spaces are $M_{r}^{R}=\operatorname{Hom}_{H}\left(V_{r}, V_{R}\right)$. Since the space $\operatorname{Inv}_{r, r^{\prime}}$ of vectors in $V_{r} \otimes V_{r^{\prime}}$ invariant under the diagonal action of $H$ is canonically isomorphic to $\delta_{\bar{r}, r^{\prime}} \mathbb{C}$, the decomposition (3.4) reduces to

$$
L^{2}(G)_{H} \cong \bigoplus_{R, r} M_{r}^{R} \otimes \bar{M}_{r}^{R}
$$

Let $\mathbf{h}^{\perp}$ be the orthogonal complement of $\mathbf{h}$ in $\mathbf{g}$. We shall choose the basis $\left(t^{A}\right)=\left(\left(t^{\mathbf{a}}\right),\left(t^{\alpha}\right)\right)$ of $\mathrm{g}$ so that $t^{\mathrm{a}}\left(t^{\alpha}\right) \operatorname{span} \mathrm{h}\left(\mathrm{h}^{\perp}\right)$. Both $-\Delta_{G} \equiv J^{A \pm} J^{A \pm}$ and $-\Delta_{H}^{ \pm} \equiv J^{\mathrm{a} \pm} J^{\mathrm{a} \pm}$ commute 
with the generators $J^{\mathrm{b} \pm}$ of the left and right $\mathrm{h}$ symmetry, so that we may take $-\frac{2}{k}\left(\Delta_{G}-\right.$ $\left.\Delta_{H}^{ \pm}\right)=\frac{2}{k} J^{\alpha \pm} J^{\alpha \pm}$ as the Hamiltonian of the reduced system (both signs give the same operator on $\left.L^{2}(G)_{H}\right)$. Finally, we may take the algebra $C^{\infty}(G)_{H}$ of multiplication by the functions invariant under the adjoint action of $H$ and consider the triple

$$
\left(L^{2}(G)_{H},-\frac{2}{k}\left(\Delta_{G}-\Delta_{H}^{ \pm}\right), C^{\infty}(G)_{H}\right)
$$

representing what we shall, somewhat abusively, call the coset geometry.

Is the coset geometry a standard Riemannian one? This is not so and there are two reasons for it. First, since the adjoint action is not free, $G / \operatorname{Ad}(H)$ might not be a smooth manifold. In particular, for $H=G, L^{2}(G)_{G}$ is the space of class functions on $G$ spanned by the characters of irreducible representations. $G / \operatorname{Ad}(G)$ may be identified with the orbifold $T / W$, where $T$ is the Cartan subgroup of $G$ and $W$ is the Weyl group. Second, $-\frac{2}{k}\left(\Delta_{G}-\Delta_{H}^{ \pm}\right)$differs from ( $-\frac{1}{2}$ times) the Laplace-Beltrami operator for the (in general singular) metric $\gamma$ on $G / \operatorname{Ad}(H)$ which may be extracted from the triple (3.25) by the procedure described in Introduction. The two operators are, essentially, different quantizations of the same classical energy of a geodesic motion on $G / \operatorname{Ad}(H)$. Let us explain the last point in more details. A similar situation in the context of gauge theories has been analyzed in [21].

The quantum mechanical system (3.25) may be obtained by quantization of a classical one which couples the geodesic motion of the particle on the group $G$ to $\mathbf{h}$-valued gauge fields $\tau \mapsto A_{ \pm}(\tau)$. The action functional for the coupled system is

$$
\begin{aligned}
S\left(g(\cdot), A_{ \pm}(\cdot)\right)= & -\frac{k}{4} \int \operatorname{tr}\left(g^{-1} \partial_{\tau} g\right)^{2} d \tau \\
& +\frac{k}{2} \int \operatorname{tr}\left(\left(g \partial_{\tau} g^{-1}\right) A_{-}+A_{+}\left(g^{-1} \partial_{\tau} g\right)+g A_{+} g^{-1} A_{-}-A_{+} A_{-}\right) d \tau
\end{aligned}
$$

and is invariant under the gauge transformations $g \mapsto h g h^{-1}, A_{ \pm} \mapsto h A_{ \pm} h^{-1}+h \partial_{\tau} h^{-1}$ for arbitrary $H$-valued $h(\tau)$. Note that the gauge field enters quadratically and may be easily eliminated in the functional integral leading to the effective action [24]

$$
\begin{aligned}
S_{\mathrm{eff}}(g(\cdot))=-\frac{k}{4} \int & \operatorname{tr}\left[\left(g^{-1} \partial_{\tau} g\right)\left(g^{-1} \partial_{\tau} g\right)\right. \\
+ & \left.2\left(g^{-1} \partial_{\tau} g\right)\left(1-E \operatorname{Ad}_{g} E\right)^{-1} E \operatorname{Ad}_{g}\left(g^{-1} \partial_{\tau} g\right)\right] d \tau,
\end{aligned}
$$

where $E$ is the orthogonal projection in $\mathbf{g}$ onto $\mathbf{h}$ and $E^{\perp}=1-E$. The effective action is invariant under the transformations $g \mapsto h g h^{-1}$, again with $h(\tau)$ taking values in $H$, so that it defines the geodesic flow on $G / \operatorname{Ad}(H)$ with respect to a (in general singular) metric which may be easily read off (3.27). Viewing $\frac{1}{i} g^{-1} \partial_{\tau} g \equiv X$ modulo $\operatorname{Ad}_{g^{-1}}(Y)-Y$ with $Y \in \mathbf{h}$ as representing vectors tangent to $G / \operatorname{Ad}(H)$ at point $[g]$, we obtain for their length squared

$$
\|X\|^{2}=\frac{k}{2} \operatorname{tr}\left[X^{2}+2 X\left(1-E \operatorname{Ad}_{g} E\right)^{-1} E \operatorname{Ad}_{g} X\right]
$$

The dual metric on the cotangent bundle of $G / \operatorname{Ad}(H)$ is given by a simpler expression. If $X^{\prime} \in \mathrm{g}$ s.t. $E\left(\operatorname{Ad}_{g}\left(X^{\prime}\right)-X^{\prime}\right)=0$ represents a covector $\frac{1}{i} \operatorname{tr} X^{\prime} g^{-1} d g$ tangent to $G / \operatorname{Ad}(H)$ at 
$[g]$ then

$$
\left\|X^{\prime}\right\|^{2}=\frac{2}{k} \operatorname{tr}\left(E^{\perp} X^{\prime}\right)^{2}=\frac{2}{k}\left(\operatorname{tr} X^{\prime 2}-\operatorname{tr} X^{\prime} E X^{\prime}\right)
$$

which is the classical version of the quantum expression $-\frac{2}{k}\left(\Delta_{G}-\Delta_{H}^{-}\right)$for the coset Hamiltonian. In fact, classically, the coset mechanics is the Hamiltonian reduction [29] of the particle on $G$ with Hamiltonian (3.29) by the adjoint action of $H$. Notice that vanishing of $E\left(\operatorname{Ad}_{g}\left(X^{\prime}\right)-X^{\prime}\right)$ implies that

$$
E X^{\prime}=\left(1-E \operatorname{Ad}_{g} E\right)^{-1} E \operatorname{Ad}_{g} E^{\perp} X^{\prime}
$$

so that we may parametrize the covectors tangent to $G / \operatorname{Ad}(H)$ by elements $E^{\perp} X^{\prime} \in \mathbf{h}^{\perp}$. In particular, the (imaginary) covectors $\psi^{\alpha-}$ corresponding to $E^{\perp} X^{\prime}=2 i \sqrt{\frac{k}{2}} t^{\alpha}$ span the space cotangent vectors to $G / \operatorname{Ad}(H)$ at point $[g]$. Explicitly,

$$
\psi^{-} \equiv t^{\alpha} \psi^{\alpha-}=\sqrt{\frac{k}{2}} E^{\perp}\left(g^{-1} d g+\operatorname{Ad}_{g^{-1}}\left(1-E \operatorname{Ad}_{g^{-1}} E\right)^{-1} E\left(g^{-1} d g\right)\right)
$$

Changing $g \mapsto g^{-1}$ we obtain another basis

$$
\psi^{+} \equiv t^{\alpha} \psi^{\alpha+}=\sqrt{\frac{k}{2}} E^{\perp}\left((d g) g^{-1}+\operatorname{Ad}_{g}\left(1-E \operatorname{Ad}_{g} E\right)^{-1} E\left((d g) g^{-1}\right)\right)
$$

The $\mathbf{h}^{\perp}$-valued 1-forms (3.31) and (3.32) transform covariantly under the adjoint action of $H$ on $G$ and vanish on vectors tangent to the orbits of that action. As we shall see below in Sect. 4.5 , they appear naturally in the supersymmetric version of the coset mechanics. Due to the simple form (3.29) of the dual metric, also the tangent spaces to $G / \operatorname{Ad}(H)$ may be identified with $\mathbf{h}^{\perp}$.

Let $d[g]$ denote the volume element on $G / \operatorname{Ad}(H)$ determined by the metric (3.28). There exists a (possibly singular) function $\sigma$ on $G / \operatorname{Ad}(H)$ such that for $f \in L^{2}(G)_{H}$,

$$
\int_{G}|f|^{2} d g=\int_{G / \operatorname{Ad}(H)}|f|^{2} \mathrm{e}^{\sigma} d[g]
$$

( $\mathrm{e}^{\sigma}$ measures, in a sense, a volume of orbits of the adjoint action of $H$ ). Now

$$
\begin{aligned}
\left\langle f\left|-\frac{2}{k}\left(\Delta_{G}-\Delta_{H}\right)\right| f\right\rangle & =\frac{2}{k} \int_{G}\left(\left|J^{A} f\right|^{2}-\left|J^{\mathrm{a}} f\right|^{2}\right) d g \\
& =\frac{1}{2} \int_{G / \operatorname{Ad}(H)}\|d f\|^{2} \mathrm{e}^{\sigma} d[g],
\end{aligned}
$$

where $\|d f([g])\|^{2}$ is given by eq. (3.29). As we see, $-\frac{2}{k}\left(\Delta_{G}-\Delta_{H}\right)$ differs from ( $-\frac{1}{2}$ times) the Laplace-Beltrami operator on $G / \operatorname{Ad}(H)$ for metric (3.28) by the replacement of the Riemannian volume $d v_{\gamma}$ by $\mathrm{e}^{\sigma} d v_{\gamma}$, both in the definition of the $L^{2}$ scalar product and in the Dirichlet form. This has been noticed in [54], see also [16], for a specific non-compact coset. The function $\sigma$ is 
often called a "dilaton" in the physical literature. It is a quantum-mechanical effect: it adds a correcting potential of order $\frac{1}{k^{2}}$ to the Laplace-Beltrami operator [21].

\subsection{Coset CFT models}

The coset construction is the field theory version of the quantum-mechanical reduction by $H \subset G_{\text {diag }} \subset G \times G$. The original idea of [28] was to decompose a representation of the affine Kac-Moody algebra $\hat{\mathbf{g}}$ with respect to a subalgebra $\widehat{\mathbf{h}}$ and to look at the multiplicity spaces. If $\mathbf{V}_{R}^{k}$ carries the irreducible unitary representation of $\hat{\mathbf{g}}$ then

$$
\mathrm{V}_{R}^{k} \cong \bigoplus_{\substack{r \\ \text { integrable }}} \mathbf{M}_{r}^{k, R} \otimes \mathbf{V}_{r}^{k}
$$

where $\mathbf{V}_{r}^{k}$ are the spaces of irreducible unitary representations of $\hat{\mathbf{h}}$. The key point is that the multiplicity spaces $\mathbf{M}_{r}^{k, R}$ carry unitary representations of the Virasoro algebra obtained by taking the difference of the two Sugawara constructions, relative to $\widehat{\mathbf{g}}$ and relative to $\widehat{\mathbf{h}}$ :

$$
L_{n}^{c s \pm}=\frac{1}{k+g^{\vee}} \sum_{m}: J_{m}^{A \pm} J_{n-m}^{A \pm}:-\frac{1}{k+h^{\vee}} \sum_{m}: J_{m}^{\mathrm{a} \pm} J_{n-m}^{\mathrm{a} \pm}: \text {. }
$$

The operators $L_{n}^{\mathrm{cs} \pm}$ commute with the currents $J_{n}^{\mathrm{b} \pm}$ so that, indeed, they act in $\mathbf{M}_{r}^{k, R}$. They form a representation of the Virasoro algebra with central charge $c^{G / H} \equiv c_{k}^{G}-c_{k}^{H}$, the difference of the two Sugawara central charges.

The Hilbert space of the coset theory is the (norm completed) left-right combination of the multiplicity spaces $\mathrm{M}_{r}^{k, R}$ :

$$
\mathbb{H}=\left\{\bigoplus_{\substack{R, r \\ \text { integrable }}} \mathbf{M}_{r}^{k, R} \otimes \mathbf{M}_{\bar{r}}^{k, \bar{R}}\right\}^{-}
$$

Equivalently, we may set

$$
\left.\mathbb{H}=\left\{|\phi\rangle \in \mathbb{H}_{\mathrm{wzw}}\left|J_{n}^{\mathrm{at}}\right| \phi\right\rangle=0 \text { for } n>0,\left(J_{0}^{\mathrm{a}+}+J_{0}^{\mathrm{a}-}\right)|\phi\rangle=0\right\},
$$

where $\mathbb{H}_{\mathrm{wZW}}$ denotes the space of states of the group $G$ level $k$ WZW model. $\mathcal{H}=L_{0}^{\text {cs }}+$ $L_{0}^{\mathrm{cs}-}-\frac{1}{12} c^{G / H}$ defines the Hamiltonian on $\mathbb{H}$.

To each element $|\phi\rangle \in \mathbb{H}$ annihilated by $L_{n}^{\text {cst }}$ for $n>0$ there corresponds a primary field (out of a local family) $V_{|\phi\rangle}^{\text {cs }}\left(z_{ \pm}\right)$of the Virasoro algebras $\left(L_{n}^{\text {cs }} \pm\right)$ with the property that

$$
\lim _{z_{ \pm} \rightarrow 0} V_{|\phi\rangle}^{\mathrm{cs}}\left(z_{ \pm}\right)|\mathrm{vac}\rangle=|\phi\rangle
$$

Taking the algebra $\mathcal{A}$ generated by these primary fields, we obtain the triple $(\mathbb{H}, \mathcal{H}, \mathcal{A})$ characterizing the geometry of the coset CFT.

On the Lagrangian level, the coset construction corresponds to gauging of a subgroup $H$ of the global $G \times G$ symmetry in the WZW theory [3][23][24][39]. Most of the subgroups would 
lead to gauge anomalies, but $H \subset G_{\text {diag }}$ do not. In the diagonal case, the gauged WZW action is given by the formula

$$
\begin{aligned}
& S\left(g(\cdot), A_{ \pm}(\cdot)\right)=S_{\mathrm{WZW}}(g) \\
& +\frac{k}{4 \pi} \int \operatorname{tr}\left(\left(g \partial_{+} g^{-1}\right) A_{-}+A_{+}\left(g^{-1} \partial_{-} g\right)+g A_{+} g^{-1} A_{-}-A_{+} A_{-}\right) d \sigma d \tau,
\end{aligned}
$$

where $\partial_{ \pm}=\partial_{\tau} \pm \partial_{\sigma}$ and $A_{ \pm}(\sigma, \tau)$ are the components of an $\mathbf{h}$-valued gauge field which, again, enters quadratically and may be eliminated from the functional integral.

The above presentation of the coset models suggests a natural way to associate to it an effective target geometry. As the small space of states we may take

$$
\left.\mathbb{H}_{0}=\left\{|\phi\rangle \in \mathbb{H}_{\mathrm{wzw}}\left|J_{n}^{A \pm}\right| \phi\right\rangle=0 \text { for } n>0,\left(J_{0}^{\mathrm{a}+}+J_{0}^{\mathrm{a}-}\right)|\phi\rangle=0\right\}
$$

which is defined using the current algebra. Notice that $\mathbb{H}_{0} \cong L_{k}^{2}(G)_{H}$ which is the subspace of $L^{2}(G)_{H}$ obtained by summing in (3.24) only over the representations integrable at level $k$. The restricted Hamiltonian

$$
\mathcal{H}_{0}=\left.\left(\mathcal{H}+\frac{1}{12} c^{G / H}\right)\right|_{\mathbb{H}_{0}}=-\frac{2}{k+g^{\vee}} \Delta_{G}+\frac{2}{k+h^{\vee}} \Delta_{H}^{ \pm}
$$

Let $E_{0}$ denote, as usual, the orthogonal projection onto $\mathbb{H}_{0}$. We may generate the small algebra $\mathcal{A}_{0}$ by operators $\left.E_{0} V_{|\phi\rangle}^{\mathrm{cs}}(1)\right|_{\mathbb{F}_{0}}$ for $|\phi\rangle \in \mathbb{H}_{0}$. Their action may be explicitly described in the following way. Let $f_{l}, l=1,2,3$, be three functions in $M_{r_{i}}^{R_{i}} \otimes M_{r_{i}}^{R_{i}} \subset L_{k}^{2}(G)_{H}$, see (3.24). Recall that $M_{r}^{R}=\operatorname{Hom}_{H}\left(V_{r}, V_{R}\right) \subset \operatorname{Hom}\left(V_{r}, V_{R}\right)$. Let $C_{\left(R_{1}\right)}^{k}$ be the vector of the operator product coefficients in $\operatorname{Inv}_{\left(R_{l}\right)} \otimes \operatorname{Inv}_{\left(\bar{R}_{l}\right)}$ giving the matrix elements of the group $G$ primary fields, see (3.18), and $c_{\left(r_{l}\right)}^{k} \in \operatorname{Inv}_{\left(r_{l}\right)} \otimes \operatorname{Inv}_{\left(\bar{r}_{l}\right)}$ the one for the group $H$. Let $\tilde{c}_{\left(r_{l}\right)}^{k} \in \operatorname{Inv}_{\left(r_{l}\right)} \otimes \operatorname{Inv}_{\left(\bar{r}_{l}\right)}$ be the element obtained by inverting $c_{\left(r_{l}\right)}^{k}$ viewed as an element of $\operatorname{End}\left(\operatorname{Inv}\left(r_{l}\right)\right)$ on its image. $\tilde{c}_{\left(r_{l}\right)}^{k}$ is set to zero on the kernel of $c_{\left(r_{l}\right)}^{k}$ which is orthogonal to its image. Matrix elements of the fields $V_{|\phi\rangle}^{\mathrm{cs}}(1)$ between states in $\mathbb{H}_{0}$ are given by

$$
\left\langle\overline{f_{3}}\left|V_{\left|f_{2}\right\rangle}^{\mathrm{cs}}(1)\right| f_{1}\right\rangle=\left\langle C_{\left(R_{l}\right)}^{k}\right|\left(\otimes\left(f_{l}\right)\left(\tilde{c}_{\left(r_{l}\right)}^{k}\right)\right\rangle \text {, }
$$

where $\otimes_{l} f_{l}$ is viewed as an element of $\operatorname{Hom}\left(\left(\otimes_{l} V_{r_{l}}\right) \otimes\left(\otimes_{l} V_{\bar{r}_{l}}\right),\left(\otimes_{l} V_{R_{l}}\right) \otimes\left(\otimes_{l} V_{\bar{R}_{l}}\right)\right)$. Eq. (3.22) implies then that

$$
\lim _{k \rightarrow \infty}\left\langle\overline{f_{3}}\left|V_{\left|f_{2}\right\rangle}^{\mathrm{cs}}(1)\right| f_{1}\right\rangle=\int_{G} \prod_{l} f_{l}(g) d g
$$

Hence $\left(\mathbb{H}_{0}, \mathcal{H}_{0}, \mathcal{A}_{0}\right)$ encodes a finite non-commutative geometry deforming the coset geometry given by the triple (3.25). Again the deformation disappears in the classical limit $k \rightarrow \infty$.

In the special case when $H=G, L^{2}(G)_{G}$ is the space of class functions on $G$. We may take $f_{l}$ to be the characters $\chi_{l}$ of integrable representations $R_{l}$. It is easy to see then from (3.41) that

$$
\left\langle\overline{\chi_{3}}\left|V_{\left|\chi_{2}\right\rangle}^{\mathrm{cs}}(1)\right| \chi_{1}\right\rangle=\operatorname{rank}\left(C_{\left(R_{l}\right)}^{k}\right)
$$


where $C_{\left(R_{l}\right)}^{k}$ is viewed as an element of $\operatorname{End}\left(\operatorname{Inv}_{\left(R_{l}\right)}\right)$. These are the so called Verlinde dimensions [48] which in the limit $k \rightarrow \infty$ tend to the dimensions of $\operatorname{Inv}_{\left(R_{l}\right)}$.

One may envisage an alternative procedure, based on the coset Virasoro algebra rather than on the current algebra, for extracting the effective target geometry from the coset theory. It would be based on the following small Hilbert space :

$$
\mathbb{H}_{0}^{\prime}=\left\{|\phi \in \mathbb{H}| L_{n}^{\mathrm{cs} \pm}|\phi\rangle=0 \text { for } n>0,\left(L_{0}^{\mathrm{cs}+}-L_{0}^{\mathrm{cs}-}\right)|\phi\rangle=0\right\}
$$

with the small Hamiltonian $\mathcal{H}_{0}^{\prime}=\left.\left(\mathcal{H}+\frac{1}{12} c^{G / H}\right)\right|_{\mathbb{P}_{0}^{\prime}}$. The small algebra $\mathcal{A}_{0}^{\prime}$ will then begenerated by operators $\left.E_{0}^{\prime} V_{|\phi\rangle}^{\mathrm{cs}}(1)\right|_{\mathbb{H}_{0}^{\prime}}$ with $|\phi\rangle \in \mathbb{H}_{0}^{\prime}$. Notice that $\mathbb{H}_{0} \subset \mathbb{H}_{0}^{\prime}$.

The classical example of a coset theory [28] is obtained by choosing $G=S U(2) \times S U(2)$ with $H$ equal to the diagonal $S U(2)$. Since $G$ is not simple, the coupling constants for the WZW theory may be chosen independently for both $S U(2)$ factors and one sets them to $k$ and to 1 , respectively. The coset models obtained this way give the unitary theories in the series of minimal models of [4]. The Hilbert space of these theories is the diagonal combination of the irreducible unitary representations [20] of the Virasoro algebras $\left(L_{n}^{\text {cs }}\right)$ with central charge $c_{k+2}^{\min }=1-\frac{6}{(k+2)(k+3)} . \quad S U(2) \times S U(2) \ni\left(g_{1}, g_{2}\right) \mapsto \operatorname{tr} g_{1}$ defines a simple function $f$ on $S U(2) \times S U(2)$ invariant under the adjoint action of the diagonal $S U(2)$ subgroup. The corresponding primary coset field $V_{|f\rangle}^{\text {cs }}\left(z_{ \pm}\right)$, labeled $\phi_{22}\left(z_{ \pm}\right)$in [4], has conformal weights $\Delta_{22}^{ \pm}=\frac{3}{4(k+2)(k+3)}$. An interesting question is what is the limit of the effective target geometries $\left(\mathbb{H}_{0}^{\prime}, \mathcal{H}_{0}^{\prime}, \mathcal{A}_{0}^{\prime}\right)$ for the minimal models when $k \rightarrow \infty$ ?

Arguments have been advanced [56][57] in support of the conjecture that Green's functions of $\phi_{22}$, for which exact expressions in terms of finite-dimensional integrals are known ${ }^{7}$, coincide with the scaling limit of Green's functions of the field $\varphi$ in critical $P(\varphi)_{2}$ models, with $P$ of degree $2 k+2$. For $\mathrm{k}=1$, one obtains this way the scaling limit of Green's functions of the two-dimensional critical Ising model with critical exponent $\eta=4 \Delta_{22}^{ \pm}=\frac{1}{4}$. The model with $k=2$ should correspond to the tricritical Ising model for which one obtains $\eta=4 \Delta_{22}^{ \pm}=\frac{3}{20}$. It is remarkable that, although detailed rigorous control of these scaling limits has long eluded the attempts of constructive field theory, we apparently have at our disposal the exact expressions for the critical exponents and even for limiting Green's functions. It may be worthwhile to produce a clean proof that one obtains this way an example of a theory satisfying all (massless) quantum field theory axioms.

\section{Supersymmetric CFT}

\subsection{Geodesic motion of a superparticle}

In Sects. 1, 3.1 and 3.3, we have sketched how the Riemannian geometry of a manifold $M$ is reflected in the Laplace-Beltrami operator acting in $L^{2}(M)$. For many purposes, however,

\footnotetext{
${ }^{7}$ the 4-point function, for example, is a bilinear combination of the hypergeometric functions
} 
especially for constructing characteristic classes of the manifold and for analyzing geometry with torsion, it is more convenient to work with the Dirac operator $\not \partial$ acting in the space of sections of the spinor bundle over $M$. A suitable framework may be obtained by quantizing a supersymmetric (SUSY) version of the geodesic motion on $M$. Instead of trajectories $\tau \mapsto$ $x(\tau)$, one considers

$$
\mathbf{x}^{\mu}\left(\tau, \theta^{ \pm}\right) \equiv x^{\mu}(\tau)+\theta^{+} \psi^{\mu+}(\tau)+\theta^{-} \psi^{\mu-}(\tau)+\theta^{+} \theta^{-} F^{\mu}(\tau)
$$

where $x^{\mu}(\cdot), F^{\mu}(\cdot)$ are functions and $\psi^{\mu \pm}(\tau)$ as well as $\theta^{ \pm}$are anticommuting Grassmann generators. We could also consider a system with only one $\theta$ parameter. The pair $\theta^{ \pm}$is inherited from the left-right moving sectors of the 1+1-dimensional SUSY sigma model if we restrict it to fields constant in space, as explained below. With the use of operators

$$
D_{ \pm} \equiv \partial_{\theta^{ \pm}}+i \theta^{ \pm} \partial_{t}
$$

the action functional for the geodesic motion of a superparticle on $M$ may be written as

$$
S(\mathbf{x}(\cdot))=\frac{1}{2} \int \gamma_{\mu \nu}(\mathbf{x})\left(D_{+} \mathbf{x}^{\mu}\right)\left(D_{-} \mathbf{x}^{\nu}\right) d \tau d \theta^{+} d \theta^{-},
$$

where the Berezin integration over $\theta^{ \pm}$is defined by the standard rule $\int d \theta^{ \pm}=0, \int \theta^{ \pm} d \theta^{ \pm}=1$. It will be convenient to add to the action(4.3) the term

$$
S^{\prime}(\mathbf{x}(\cdot))=\frac{1}{2} \int \beta_{\mu \nu}(\mathbf{x})\left(D_{+} \mathbf{x}^{\mu}\right)\left(D_{-} \mathbf{x}^{\nu}\right) d \tau d \theta^{+} d \theta^{-}
$$

with $\beta_{\mu \nu} d x^{\mu} \wedge d x^{\nu} \equiv \beta$ a 2-form on $M$. Performing the $\theta^{ \pm}$integration (compare Sect. 23.1b of [49]), one obtains the component expression for $S^{\text {tot }} \equiv S+S^{\prime}$ :

$$
\begin{array}{r}
S^{\mathrm{tot}}(\mathbf{x}(\cdot))=\int\left[\frac{1}{2} \gamma_{\mu \nu}\left(\partial_{t} x^{\mu}\right)\left(\partial_{t} x^{\nu}\right)+\frac{i}{2} \gamma_{\mu \nu} \psi^{\mu+} \nabla_{t}^{+} \psi^{\nu+}+\frac{i}{2} \gamma_{\mu \nu} \psi^{\mu-} \nabla_{t}^{-} \psi^{\nu-}\right. \\
\left.+\frac{1}{4} R^{-}{ }_{\kappa \mu \lambda \nu} \psi^{\kappa+} \psi^{\mu+} \psi^{\lambda-} \psi^{\nu-}-\gamma_{\rho \sigma}\left(F^{\rho}-\Gamma^{-\rho}{ }_{\mu \nu} \psi^{\mu+} \psi^{\nu-}\right)\left(F^{\sigma}-\Gamma^{-\sigma}{ }_{\kappa \lambda} \psi^{\kappa+} \psi^{\lambda-}\right)\right] d \tau,
\end{array}
$$

where

$$
\nabla_{t}^{ \pm} \psi^{\mu \pm}=\partial_{t} \psi^{\mu \pm}+\Gamma_{\kappa \lambda}^{ \pm \mu}\left(\partial_{t} x^{\kappa}\right) \psi^{\lambda \pm}
$$

are the covariant derivatives with respect to connections with torsion,

$$
\Gamma_{\kappa \lambda}^{ \pm \mu}=\Gamma_{\kappa \lambda}^{\mu} \pm \frac{1}{2} \gamma^{\mu \nu} H_{\nu \kappa \lambda},
$$

where

$$
\Gamma_{\kappa \lambda}^{\mu}=\frac{1}{2} \gamma^{\mu \nu}\left(\partial_{\kappa} \gamma_{\nu \lambda}+\partial_{\lambda} \gamma_{\nu \kappa}-\partial_{\nu} \gamma_{\kappa \lambda}\right), \quad H_{\nu \kappa \lambda}=\partial_{\nu} \beta_{\kappa \lambda}+\partial_{\kappa} \beta_{\lambda \nu}+\partial_{\lambda} \beta_{\nu \kappa}
$$

$\left(\Gamma^{\mu}{ }_{\kappa \lambda}\right)$ define the Levi-Civita connection, while $\Gamma^{ \pm \mu}{ }_{\kappa \lambda}$ is modified by a torsion term coming from the 2 -form $\beta$. Note that $\beta$ enters the above formulae only through the torsion form $d \beta=\frac{1}{3} H_{\nu \kappa \lambda} d x^{\nu} \wedge d x^{\kappa} \wedge d x^{\lambda}$.

$$
R_{\kappa \mu \lambda \nu}^{ \pm}=\partial_{\kappa} \Gamma_{\lambda \mu \nu}^{ \pm}-\partial_{\mu} \Gamma_{\lambda \kappa \nu}^{ \pm}-\Gamma_{\rho \kappa \lambda}^{ \pm} \Gamma_{\mu \nu}^{ \pm \rho}+\Gamma_{\rho \mu \lambda}^{ \pm} \Gamma_{\kappa \nu}^{ \pm \rho}
$$


are the corresponding curvature tensors of the connections with torsion ${ }^{8}$. In particular, for $\beta_{\mu \nu}=0$, they reduce to the curvature of the Levi-Civita connection. Notice that $F^{\mu}(\tau)$ are not dynamical variables. They may be eliminated from their classical equations. The classical supersymmetry is generated by the constants of motion

$$
Q^{ \pm} \equiv \frac{1}{\sqrt{2}}\left(\mp \gamma_{\mu \nu} \partial_{t} x^{\mu} \psi^{\nu \pm}+\frac{i}{6} H_{\mu \nu \kappa} \psi^{\mu \pm} \psi^{\nu \pm} \psi^{\kappa \pm}\right)
$$

It is not difficult to quantize the system. As the Hilbert space $\mathbb{H}$ of states, we shall take $L^{2}(S \otimes S)$ for even dimensional $M$, or $L^{2}\left(S \otimes S \otimes \mathbb{C}^{2}\right)$ for odd dimensional $M$, where $S$ is the bundle of spinors (containing both chiralities in the even-dimensional case). The fibers of $S \otimes S\left(S \otimes S \otimes \mathbb{C}^{2}\right)$ support the double Dirac algebra:

$$
\left\{\Gamma^{A \pm}, \Gamma^{B \pm}\right\}=-2 \delta^{A B}, \quad\left\{\Gamma^{A \pm}, \Gamma^{B \mp}\right\}=0, \quad\left(\Gamma^{A \pm}\right)^{*}=-\Gamma^{A \pm}
$$

related to a local vielbein ${ }^{9}\left(e_{A}^{\mu}\right), e_{A}^{\mu} e_{A}^{\nu}=\gamma^{\mu \nu}, \gamma_{\mu \nu} e_{A}^{\mu} e_{B}^{\nu}=\delta_{A B}$.

$$
\psi^{\mu \pm}=\frac{1}{i \sqrt{2}} e_{A}^{\mu} \Gamma^{A \pm}
$$

will quantize the classical Grassmann generators $\psi^{\mu \pm}(0)$.

$$
\left\{\psi^{\mu \pm}, \psi^{\nu \pm}\right\}=\gamma^{\mu \nu}, \quad\left\{\psi^{\mu \pm}, \psi^{\nu \mp}\right\}=0, \quad\left(\psi^{\mu \pm}\right)^{*}=\psi^{\mu \pm} .
$$

Introducing the spin connections with torsion by

$$
\nabla_{\nu}^{ \pm} e_{B} \equiv \omega_{\nu}^{ \pm A}{ }_{B} e_{A}
$$

we shall define the left-right covariant derivative of the double index spinors $\chi$ (sections of $S \otimes S$ or of $S \otimes S \otimes \mathbb{C}^{2}$ ) by

$$
\nabla_{\mu} \chi=\partial_{\mu} \chi-\frac{1}{8} \sum_{+,-} \omega_{\mu}^{ \pm A}\left[\Gamma^{A \pm}, \Gamma^{B \pm}\right] \chi
$$

The classical supersymmetry generators $Q^{ \pm}$of (4.10) give rise, on the quantum level, to two Dirac operators

$$
\not_{ \pm}=\frac{1}{\sqrt{2}}\left( \pm i \psi^{\mu \pm} \nabla_{\mu}+\frac{i}{6} H_{\mu \nu \kappa} \psi^{\mu \pm} \psi^{\nu \pm} \psi^{\kappa \pm}\right)
$$

with the following simple (although tedious to verify) algebra:

$$
\begin{aligned}
\frac{1}{2} \mathcal{H} \equiv \not_{+}^{2}=\not_{-}^{2}=-\frac{1}{4} \gamma^{\mu \nu}\left(\nabla_{\mu} \nabla_{\nu}-\Gamma_{\mu \nu}^{\kappa} \nabla_{\kappa}\right)+\frac{1}{16} R-\frac{1}{192} H_{\kappa \mu \nu} H^{\kappa \mu \nu} \\
-\frac{1}{32} R_{\mu \nu \kappa \lambda}^{-}\left[\psi^{\mu+}, \psi^{\nu+}\right]\left[\psi^{\kappa-}, \psi^{\lambda-}\right], \\
\left\{\not_{+}, \not \partial_{-}\right\}=0,
\end{aligned}
$$

\footnotetext{
8 indices are raised and lowered with the metric $\gamma$

${ }^{9}$ more exactly, to its lift to the spin bundle covering twice the bundle of vielbeine
} 
where $R$ is the scalar curvature of the Levi-Civita connection $\left(\Gamma_{\mu \nu}^{\kappa}\right)$.

\subsection{Superparticle on a group}

Among the simplest examples of superparticle motions is that on the group manifold $G$, with the action

$$
S^{\mathrm{tot}}\left(g, \psi^{ \pm}\right)=-\frac{k}{4} \int \operatorname{tr}\left(g^{-1} \partial_{t} g\right)^{2} d \tau+i \int \operatorname{tr}\left(\psi^{+} \partial_{t} \psi^{+}+\psi^{-} \partial_{t} \psi^{-}\right) d \tau,
$$

where $\psi^{ \pm}(\tau)=t^{A} \psi^{A \pm}(\tau)$ are the Lie algebra g-valued Grassmann variables. Let $e_{A}^{ \pm}$denote the right- (left-)invariant vector fields on $G$ generated by $\frac{3}{\sqrt{k}} t^{A} \in \mathrm{g}$. They yield two global vielbeine for a left-right invariant Riemannian metric on $G$. Setting $e_{A}^{\mu \pm} \psi^{A \pm} \equiv \psi^{\mu \pm}$ relates the variables $\psi^{A \pm}$ to $\psi^{\mu \pm}$ used above. The connections with torsion $\nabla^{ \pm}$preserve the vector fields $e_{A}^{ \pm}$, respectively, and, consequently, have no curvature. This explains the simplicity of eq. (4.18) as compared to (4.5). The torsion coefficients are given by

$$
e_{A}^{\kappa \pm} e_{B}^{\mu \pm} e_{C}^{\nu \pm} H_{\kappa \mu \nu}=-\frac{2}{\sqrt{k}} f^{A B C}
$$

(both signs in $e^{ \pm}$give the same expression) and the torsion form $\frac{1}{3} H_{\kappa \mu \nu} d x^{\kappa} \wedge d x^{\mu} \wedge d x^{\nu}=$ $\frac{i k}{3} \operatorname{tr}\left(g^{-1} d g\right)^{\wedge 3}$ and is only locally exact. Using the global vielbeine $\left(e_{A}^{\frac{3}{3}}\right)$ to trivialize the spinor bundles over $G$, we may identify the Hilbert space of states with

$$
\mathbb{H}=L^{2}(G) \otimes W
$$

where $W$ carries an irreducible representation of the double Dirac algebra (4.11), with $\psi^{A \pm}=$ $\frac{1}{i \sqrt{2}} \Gamma^{A \pm}$. By setting

$$
\begin{aligned}
& j^{A \pm}=\frac{1}{2 i} f^{A B C} \psi^{B \pm} \psi^{C \pm}, \\
& J^{A \pm}= \pm \frac{i \sqrt{k}}{2} \nabla_{e_{A}^{ \pm}}
\end{aligned}
$$

we obtain four commuting representations of the Lie algebra $\mathbf{g}$ acting in $\mathbb{H}$ :

$$
\left[j^{A \pm}, j^{B \pm}\right]=i f^{A B C} j^{C \pm}, \quad\left[J^{A \pm}, J^{B \pm}\right]=i f^{A B C} J^{C \pm} .
$$

The Dirac operators are

$$
\partial_{ \pm}=\sqrt{\frac{2}{k}}\left(\psi^{A \pm} J^{A \pm}-\frac{i}{6} f^{A B C} \psi^{A \pm} \psi^{B \pm} \psi^{C \pm}\right)
$$

and for the Hamiltonian one obtains

$$
\frac{1}{2} \mathcal{H}=\not_{ \pm}^{2}=\frac{1}{k} J^{A \pm} J^{A \pm}+\frac{g^{\vee} \operatorname{dim}(G)}{24 k} .
$$

Notice that $\not_{ \pm}^{2}$ is a strictly positive operator:

$$
\partial_{ \pm}^{2} \geq \frac{g^{\vee} \operatorname{dim}(G)}{24 k} .
$$




\subsection{Elements of the non-commutative de Rham calculus}

Suppose, for simplicity, that $M$ is compact. Given the algebra $\mathcal{A}=C^{\infty}(M)$ and a Dirac operator $\not \partial=\not_{ \pm}$, one may rewrite the de Rham differential form calculus, following Connes et al. [12][15][13], in terms of that of operators acting in the Hilbert space $H=L^{2}(S \otimes S)$ (or $L^{2}\left(S \otimes S \otimes \mathbb{C}^{2}\right)$, for $M$ odd-dimensional). This reformulation carries over to the noncommutative setting where $\not \partial$ is a selfadjoint operator on a Hilbert space $H$ and $\mathcal{A}$ is a *-algebra of operators in $B(H)$ satisfying certain regularity assumptions [12][15][13]. A brief exposition of these ideas ${ }^{10}$ is contained in Sect. 6 of [14], see also [11]. Consider operators $\omega$ acting in $H$ and given by the expressions

$$
\omega=\sum_{j} a_{j 0}\left[\not \partial, a_{j 1}\right] \cdots\left[\partial, a_{j n}\right],
$$

where $a_{j m} \in \mathcal{A}$. The space $\Omega^{\prime n}(\mathcal{A})$ of such operators clearly forms a left $\mathcal{A}$-module. Since for $a, b \in \mathcal{A}$

$$
[\not \partial, a] b=[\not \partial, a b]-a[\not \partial, b],
$$

$\Omega^{\prime n}(\mathcal{A})$ is also a right $\mathcal{A}$-module and $\Omega^{\prime}(\mathcal{A}) \equiv \oplus_{n} \Omega^{\prime n}(\mathcal{A})$ becomes a $\mathbb{Z}$-graded algebra. Let $N^{n}(\mathcal{A})$ be composed of operators

$$
\eta=\sum_{j}\left[\not \partial, a_{j 0}\right]\left[\not \partial, a_{j 1}\right] \cdots\left[\not \partial, a_{j n-1}\right]
$$

such that $\sum_{j} a_{j 0}\left[\not \partial, a_{j 1}\right] \cdots\left[\not \partial, a_{j n-1}\right]=0$. It is not difficult to see that $N(\mathcal{A})=\oplus_{n} N^{n}(\mathcal{A})$ is a left-right $\mathbb{Z}$-graded ideal in $\Omega^{\prime}(\mathcal{A})$. Define the $\mathbb{Z}$-graded algebra $\Omega(\mathcal{A}) \equiv \Omega^{\prime}(\mathcal{A}) / N(\mathcal{A})$. Notice that $\Omega^{0}(\mathcal{A})=\Omega^{\prime 0}(\mathcal{A})=\mathcal{A}$ and $\Omega^{1}(\mathcal{A})=\Omega^{\prime 1}(\mathcal{A})=\left\{\sum_{j} a_{j 0}\left[\not \partial, a_{j 1}\right] \mid a_{j 0}, a_{j_{1}} \in \mathcal{A}\right\}$. Each $\Omega^{n}(\mathcal{A})$ is a left-right $\mathcal{A}$-module. On $\Omega(\mathcal{A})$, one may define the graded differential $d$ acting on representatives by

$$
d \sum_{j} a_{j 0}\left[\not \partial, a_{j 1}\right] \cdots\left[\not \partial, a_{j n}\right]=\sum_{j}\left[\not \partial, a_{j 0}\right]\left[\not \partial, a_{j 1}\right] \cdots\left[\not \partial, a_{j n}\right] .
$$

We have

$$
d(\omega \rho)=(d \omega) \rho+(-1)^{\operatorname{deg}(\omega)} \omega d \rho
$$

In the general setup, $\Omega^{n}(\mathcal{A})$ plays the role of the space of smooth $n$-forms.

We shall be interested in the situations where the trace of non-zero operators in $\Omega^{\prime n}(\mathcal{A})$ diverges, but one may define its regularized version (which we shall denote by the integral sign) for example by

$$
\int \omega \equiv \lim _{\epsilon \searrow 0} \frac{\operatorname{tr}\left(\omega \mathrm{e}^{-\epsilon \not^{2}}\right)}{\operatorname{tr}\left(\mathrm{e}^{-\epsilon \not^{2}}\right)}
$$

\footnotetext{
${ }^{10}$ a different approach to non-commutative de Rham calculus may be found in [17]
} 
or using the more sophisticated Dixmier trace [13], so that

$$
(\omega, \rho) \equiv \int \omega^{*} \rho
$$

defines a scalar product on $\Omega^{\prime n}(\mathcal{A})$. We may then complete $\Omega^{\prime n}(\mathcal{A})$ to a Hilbert space and embed $\Omega^{n}(\mathcal{A})$ into the subspace $\Lambda^{n}(\mathcal{A})$ of this completion perpendicular to $N(\mathcal{A}) . \Lambda^{n}(\mathcal{A})$ plays the role of the space of square integrable $n$-forms.

In the commutative example, with $H=L^{2}(S \otimes S)\left(L^{2}\left(S \otimes S \otimes \mathbb{C}^{2}\right)\right), \not \partial=\not \partial \pm$ and $\mathcal{A}=C^{\infty}(M)$

$$
\omega=( \pm i)^{n} a_{j_{0}}\left(\partial_{\mu_{1}} a_{j 1}\right) \cdots\left(\partial_{\mu_{n}} a_{j n}\right) \psi^{\mu_{1} \pm} \cdots \psi^{\mu_{n} \pm}
$$

( \pm depending on whether $\not \partial=\not_{+}$or $\not \partial=\not_{-}$). The class of $\omega$, represented by $\omega^{\perp} \in \Lambda^{n}(\mathcal{A})$, may be identified with the de Rham n-form

$$
\tilde{\omega}=a_{j_{0}}\left(\partial_{\mu_{1}} a_{j 1}\right) \cdots\left(\partial_{\mu_{n}} a_{j_{n}}\right) d x^{\mu_{1}} \wedge \ldots \wedge d x^{\mu_{n}} .
$$

The scalar product $\left(\omega^{\perp}, \rho^{\perp}\right)$ is then proportional to $\int_{M} * \tilde{\omega} \wedge \tilde{\rho}$, where $* \tilde{\omega}$ is the Hodge star of $\tilde{\omega}$.

In the general setup, one defines a vector bundle $E$ over $\mathcal{A}$ as a finitely generated, projective $^{11}$ left $\mathcal{A}$-module. A connection on $E$ is defined as a linear mapping $\nabla: E \rightarrow \Omega^{1}(\mathcal{A}) \otimes_{\mathcal{A}} E$ such that, for $a \in \mathcal{A}$ and $s \in E$,

$$
\nabla(a s)=d a \otimes s+a \nabla s
$$

$\nabla$ may be uniquely extended to an endomorphism of the graded left $\Omega(\mathcal{A})$-module $\Omega(\mathcal{A}) \otimes_{\mathcal{A}} E$ satisfying

$$
\nabla(\omega \phi)=(d \omega) \phi+(-1)^{\operatorname{deg}(\omega)} \omega \nabla \phi
$$

One may then define the curvature $R(\nabla)$ by

$$
R(\nabla)=-\left.\nabla^{2}\right|_{E}
$$

$R(\nabla): E \rightarrow \Omega^{2}(\mathcal{A}) \otimes_{\mathcal{A}} E$ and obeys $R(\nabla)(a s)=a R(\nabla) s$ for $a \in \mathcal{A}$ and $s \in E$, i.e. it is an $\mathcal{A}$-tensor $[11]$.

If the left $\mathcal{A}$-module $\Omega^{1}(\mathcal{A})$ is finitely generated and projective, we may view it as the cotangent bundle. For a connection $\nabla: \Omega^{1}(\mathcal{A}) \rightarrow \Omega^{1}(\mathcal{A}) \otimes_{\mathcal{A}} \Omega^{1}(\mathcal{A})$ one may define noncommutative torsion [11] as the map $T(\nabla): \Omega^{1}(\mathcal{A}) \rightarrow \Omega^{2}(\mathcal{A})$ given by

$$
T(\nabla)=d-m \nabla,
$$

where $m: \Omega^{1}(\mathcal{A}) \otimes_{\mathcal{A}} \Omega^{1}(\mathcal{A}) \rightarrow \Omega^{2}(\mathcal{A})$ is the multiplication map. It follows that $T(\nabla)(a \omega)=$ $a T(\nabla) \omega: T(\nabla)$ is an $\mathcal{A}$-tensor. Also the notions of Levi-Civita connection, Ricci curvature and scalar curvature may be introduced in the non-commutative setup [11].

\footnotetext{
11 i.e. the image of an idempotent in a finite free module
} 
In the commutative case, the above definition of (finite-dimensional) vector bundles coincides with the standard one if we represent a vector bundle by the module of its smooth sections. In particular, commutative $\Omega^{1}(\mathcal{A})$ is a projective, finitely generated left(-right) $\mathcal{A}$-module representing the cotangent bundle and the covariant derivative $\nabla_{\mu}$ given by (4.15) allows to define a linear map $\nabla: \Omega^{1}(\mathcal{A}) \rightarrow \Omega^{1} \otimes_{\mathcal{A}} \Omega^{1}(\mathcal{A})$ by

$$
\nabla \omega=\sum_{j} a_{j} \psi^{\mu} \otimes\left[\nabla_{\mu}, \omega\right]
$$

where $\left(a_{j}\right)$ is a partition of unity subordinate to a covering of $M$ by coordinate charts. $\psi^{\mu}=$ $\psi^{\mu+}$ or $\psi^{\mu-}$. The commutator is that of the operators acting on sections of $S \otimes S$ (or of $S \otimes S \otimes \mathbb{C}^{2}$ ). Clearly, $\nabla$ satisfies (4.31) and represents the connections with torsion given locally by (4.7). Its curvature, defined by eq. (4.33), may easily be seen to correspond to the standard curvature 2-form. In particular, if $M=G$, as in Sect. 4.2 , then $\Omega^{1}(\mathcal{A})$ is a free $\mathcal{A}$-module with a basis $\left(\psi^{A \pm}\right)$. Setting $\psi^{ \pm} \equiv t^{A} \psi^{A \pm}$, we may identify $\sqrt{\frac{2}{k}} \psi^{+}$with the Maurer-Cartan 1-form $(d g) g^{-1}$ and $\sqrt{\frac{2}{k}} \psi^{-}$with $g^{-1} d g$. Eq. (4.35) reduces to

$$
\nabla \omega=\sqrt{\frac{2}{k}} \psi^{A \pm} \otimes\left[J^{A \pm}, \omega\right]
$$

(recall that the sign depends on whether $\not \partial=\not_{+}$or $\partial_{-}$). Consequently, $\psi^{A \pm}$ are parallel i.e. annihilated by $\nabla$ The corresponding curvature tensor vanishes. We leave as an exercise verification that the torsion $T(\nabla)$, as defined by eq. (4.34) is given by the formula

$$
T(\nabla) \psi^{A \pm}=\frac{1}{2 i} \sqrt{\frac{2}{k}} f^{A B C} \psi^{B \pm} \psi^{C \pm}
$$

Summarizing: the Riemannian geometry may be encoded in the SUSY triple $\left(L^{2}(S \otimes\right.$ $\left.\left.S\left(\otimes \mathbb{C}^{2}\right)\right), \partial_{ \pm}, C^{\infty}(M)\right)$ from which one can recover not only the metric $\gamma$ but also the de Rham calculus of differential forms which, more conveniently, may be rewritten in Connes' operator language; we have seen how the connections with the torsion 3-forms $\pm \frac{1}{3} H_{\kappa \mu \nu} d x^{\kappa} \wedge d x^{\mu} \wedge d x^{\nu}$ may be incorporated into the operator formalism of [12][15][14] whose main virtue is that it extends to non-commutative spaces characterized by general unital $*$-algebra $\mathcal{A}$.

\subsection{SUSY WZW model}

As we have seen above, the natural generalizations of the geodesic motion of a particle on a Riemannian manifold $M$ to the case of 1+1-dimensional field theory is given by the sigma model with $M$ as the target. Similarly, the geodesic motion of a superparticle generalizes to an $(\mathrm{N}=1)$ SUSY sigma model with fields $\mathbf{x}\left(\sigma, \tau, \theta^{ \pm}\right)$and an action [49]

$$
S^{\mathrm{tot}}(\mathbf{x}(\cdot))=\frac{1}{4 \pi} \int\left(\gamma_{\mu \nu}+\beta_{\mu \nu}\right)(\mathbf{x})\left(D_{+} \mathbf{x}^{\mu}\right)\left(D_{-} \mathbf{x}^{\nu}\right) d \sigma d \tau d \theta^{+} d \theta^{-},
$$

where now

$$
D_{ \pm} \equiv \partial_{\theta^{ \pm}}+i \theta^{ \pm} \partial_{ \pm}
$$


with the light-cone derivatives $\partial_{ \pm} \equiv \partial_{\tau} \pm \partial_{\sigma}$. In components,

$$
\begin{gathered}
S^{\text {tot }}(\mathbf{x}(\cdot))=\frac{1}{2 \pi} \int\left[\frac{1}{2}\left(\gamma_{\mu \nu}+\beta_{\mu \nu}\right)\left(\partial_{+} x^{\mu}\right)\left(\partial_{-} x^{\nu}\right)+\frac{i}{2} \gamma_{\mu \nu} \psi^{\mu+} \nabla_{-}^{+} \psi^{\nu+}+\frac{i}{2} \gamma_{\mu \nu} \psi^{\mu-} \nabla_{+}^{-} \psi^{\nu-}\right. \\
\left.+\frac{1}{4} R^{-}{ }_{\kappa \mu \lambda \nu} \psi^{\kappa+} \psi^{\mu+} \psi^{\lambda-} \psi^{\nu-}-\gamma_{\rho \sigma}\left(F^{\rho}-\Gamma^{-\rho}{ }_{\mu \nu} \psi^{\mu+} \psi^{\nu-}\right)\left(F^{\sigma}-\Gamma^{-\sigma}{ }_{\kappa \lambda} \psi^{\kappa+} \psi^{\lambda-}\right)\right] d \tau
\end{gathered}
$$

where

$$
\begin{aligned}
& \nabla_{+}^{ \pm} \psi^{\mu \pm}=\partial_{+} \psi^{\mu \pm}+\Gamma_{\kappa \lambda}^{ \pm \mu}\left(\partial_{+} x^{\kappa}\right) \psi^{\lambda \pm} \\
& \nabla_{-}^{ \pm} \psi^{\mu \pm}=\partial_{-} \psi^{\mu \pm}+\Gamma_{\kappa \lambda}^{ \pm \mu}\left(\partial_{-} x^{\kappa}\right) \psi^{\lambda \pm}
\end{aligned}
$$

and the supersymmetry generators

$$
Q^{ \pm} \equiv \frac{1}{\sqrt{2}}\left(\mp \gamma_{\mu \nu} \partial_{ \pm} x^{\mu} \psi^{\nu \pm}+\frac{i}{6} H_{\mu \nu \kappa} \psi^{\mu \pm} \psi^{\nu \pm} \psi^{\kappa \pm}\right)
$$

satisfy $\partial_{\mp} Q^{ \pm}=0$.

On the quantum level, the SUSY sigma model still requires renormalizations, although somewhat less severe ones than the purely bosonic model. As in the bosonic case, instead of attempting a direct construction of the models, one can use symmetry principles to obtain a rich family of exactly solvable SUSY CFT's. The simplest of them is the SUSY WZW model. It corresponds to the action

$$
S^{\text {tot }}\left(g(\cdot), \psi^{ \pm}(\cdot)\right)=S_{\text {bos }}(g)+\frac{i}{2 \pi} \int \operatorname{tr}\left(\psi^{+} \partial_{-} \psi^{+}+\psi^{-} \partial_{+} \psi^{-}\right) d \sigma d \tau
$$

where $S_{\text {bos }}(g)$ is the action of the bosonic WZW model with group $G$ and level $k-g^{\vee}$. The space of states $\mathbb{H}$ of the SUSY WZW model is the tensor product of of the space of states $\mathbb{H}_{\text {bos }}$ of the bosonic model, discussed before, (with the shifted $k$ ) and of the Fock space $\mathcal{F}$ of free Majorana-Fermi field $\psi^{ \pm}\left(z^{ \pm}\right) \equiv t^{A} \psi^{A \pm}\left(z^{ \pm}\right)$with values in the Lie algebra $\mathbf{g}$.

$$
\psi^{A \pm}\left(z_{ \pm}\right)=\sum_{n} z_{ \pm}^{-n-1 / 2} \psi_{n}^{A \pm}
$$

where the sum runs over $n \in \mathbb{Z}+\frac{1}{2}$ in the Neveu-Schwarz sector and over $n \in \mathbb{Z}$ in the Ramond sector (corresponding to two choices of the spin structure on $S^{1}$ ).

$$
\left\{\psi_{n}^{A \pm}, \psi_{m}^{B \pm}\right\}=\delta^{A B} \delta_{n+m, 0}, \quad\left\{\psi_{n}^{A+}, \psi_{m}^{B-}\right\}=0, \quad\left(\psi_{n}^{A \pm}\right)^{*}=\psi_{-n}^{A \pm} .
$$

The Neveu-Schwarz sector Fock space $\mathcal{F}^{\mathrm{NS}}$ is obtained by applying to the vacuum state $|\mathrm{vac}\rangle$ annihilated by $\psi_{n}^{A \pm}, n>0$, polynomials in $\psi_{n}^{A \pm}, n<0$ ( $n$ half-integer). The Ramond sector Fock space $\mathcal{F}^{R}$ arises by applying polynomials in $\psi_{n}^{A \pm}, n<0$, to the vector space $W$ carrying the irreducible representation of the $\psi_{0}^{A \pm}$ Clifford algebra and annihilated by $\psi_{n}^{A \pm}, n>0$ ( $n$ integer). The total fermionic Fock space of the model is

$$
\mathcal{F}=\mathcal{F}^{\mathrm{NS}} \oplus \mathcal{F}^{\mathrm{R}}
$$


It carries a representation of two commuting $\widehat{\mathrm{g}}$ current algebras given by

$$
j_{n}^{A \pm}=\frac{1}{2 i} f^{A B C} \sum_{m}: \psi_{m}^{B \pm} \psi_{n-m}^{C \pm}:
$$

and of the Virasoro algebras

$$
l_{n}^{ \pm}=-\sum_{m} \frac{1}{2} m: \psi_{m}^{A \pm} \psi_{n-m}^{A \pm}:\left(+\frac{3 \operatorname{dim}(G)}{48} \delta_{n, 0}\right)
$$

(the constant should be added in the Ramond sector). The complete space of states

$$
\mathbb{H} \equiv \mathbb{H}^{\mathrm{NS}} \oplus \mathbb{H}^{\mathrm{R}}=\left(\mathbb{H}_{\text {bos }} \otimes \mathcal{F}^{\mathrm{NS}}\right) \oplus\left(\mathbb{H}_{\text {bos }} \otimes \mathcal{F}^{\mathrm{R}}\right)
$$

carries the representation of the left-right Virasoro algebras with generators

$$
L_{n}^{ \pm}=L_{\text {bos } n}^{ \pm}+l_{n}^{ \pm}
$$

where $L_{\text {bos } n}^{ \pm}$are the Sugawara generators in $\mathbb{H}_{\text {bos }}$ constructed from the bosonic currents $J_{n}^{A \pm}$.

The total Virasoro algebras $\left(L_{n}^{ \pm}\right)$, which have central charge $c_{\text {SUSY } k}^{G}=\frac{\left(k-g^{\vee}\right) \operatorname{dim}(G)}{k}+\frac{\operatorname{dim}(G)}{2}$, may be extended to the super-Virasoro ones with additional generators

$$
Q_{n}^{ \pm}=\sqrt{\frac{2}{k}}\left(\sum_{m} \psi_{m}^{A \pm} J_{n-m}^{A}-\frac{i}{6} f^{A B C} \sum_{m, r}: \psi_{m}^{A \pm} \psi_{r}^{B \pm} \psi_{n-m-r}^{C \pm}:\right)
$$

satisfying

$$
\begin{aligned}
{\left[L_{n}^{ \pm}, Q_{m}^{ \pm}\right] } & =\left(\frac{n}{2}-m\right) Q_{n+m}^{ \pm}, \\
\left\{Q_{n}^{ \pm}, Q_{m}^{ \pm}\right\} & =2 L_{n+m}^{ \pm}+\frac{1}{3} c\left(n^{2}-\frac{1}{4}\right) \delta_{n+m, 0}
\end{aligned}
$$

where the operators $Q_{n}^{ \pm}$commute with $L_{m}^{\mp}$ and anticommute with $Q_{m}^{\mp}$. In particular,

$$
\left(Q_{0}^{ \pm}\right)^{2}=L_{0}^{ \pm}-\frac{c}{24} \quad \text { and } \quad\left\{Q_{0}^{+}, Q_{0}^{-}\right\}=0
$$

in the Ramond sector. Notice that the commutation relations (4.52) may be rewritten as the global SUSY algebra

$$
\left\{Q_{\alpha}, Q_{\beta}\right\}=\left(\gamma^{\mu} C\right)_{\alpha \beta} P_{\mu}
$$

where $\gamma^{0}=C=\left(\begin{array}{cc}0 & i \\ -i & 0\end{array}\right), \gamma^{1}=\left(\begin{array}{cc}0 & i \\ i & 0\end{array}\right)$ are two-dimensional Dirac matrices and $Q_{1} \equiv Q_{0}^{+}$, $Q_{2} \equiv Q_{0}^{-}, P_{0} \equiv \mathcal{H}=L_{0}^{+}+L_{0}^{-}-\frac{1}{12} c_{\text {susy } k}^{G}, P_{1} \equiv \mathcal{P}=L_{0}^{+}-L_{0}^{-}$. The bottom of the spectrum of the operators $Q_{\alpha}^{2}$ is attained on states $|\mathrm{vac}\rangle_{\text {bos }} \otimes w \in \mathbb{H}^{R}$, where $\mid$ vac $\rangle_{\text {bos }}$ is the bosonic vacuum in $\mathbb{H}_{\text {bos }}$ and $w \in W \subset \mathcal{F}^{\mathrm{R}}$. It is equal to $\frac{g^{v} \operatorname{dim}(G)}{24 k}>0$ (compare to (4.25)). It follows that there are no Ramond ground states (states annihilated by $Q_{0}^{ \pm}$) in the SUSY WZW model. Hence the global supersymmetry (4.53) is broken [50] and the Witten index $\operatorname{Tr}(-1)^{F}$, to which only the Ramond ground states contribute, vanishes. 
The geometry of the SUSY WZW CFT may be encoded in the triple

$$
\left(\mathbb{H}^{\mathrm{R}}, Q_{0}^{ \pm}, \mathcal{A}\right)
$$

where $\mathcal{A}$ is the bosonic algebra generated by the primary fields of the bosonic WZW model (acting trivially on the fermionic Fock space $\mathcal{F}$ ). The more general field algebra (of bosonic and fermionic operators) may be obtained by considering non-commutative differential forms

$$
\sum_{j} a_{j 0}\left[Q_{0}^{ \pm}, a_{j 1}\right] \cdots\left[Q_{0}^{ \pm}, a_{j n_{j}}\right]
$$

where $a_{j m} \in \mathcal{A}$, compare with eq. (4.26). In particular, operators $\sqrt{\frac{2}{k}} \sum_{j} a_{j 0} \sum_{n}\left[J_{-n}^{A \pm}, a_{j 1}\right] \psi_{n}^{A \pm}$ are 1 -forms.

The non-commutative geometry of the triple (4.54) is clearly infinite-dimensional and its treatment would require serious analysis. This may be avoided by restriction to the effective target geometry of the SUSY WZW model which one may define as follows. Let

$$
\left.\mathbb{H}_{0}^{\mathrm{NS}, \mathrm{R}}=\left\{|\phi\rangle \in \mathbb{H}^{\mathrm{NS}, \mathrm{R}}\left|\psi_{n}^{A \pm}\right| \phi\right\rangle=0=J_{n}^{A \pm}|\phi\rangle \text { for } n>0\right\}
$$

be the small spaces of states. Notice that

$$
\mathbb{H}_{0}^{\mathrm{NS}} \cong \mathbb{H}_{\text {bos } 0} \subset L^{2}(G), \quad \mathbb{H}_{0}^{\mathrm{R}} \cong \mathbb{H}_{\text {bos } 0} \otimes W \subset L^{2}(G) \otimes W
$$

where $\mathbb{H}_{\text {bos } 0} \cong L_{k-g^{\vee}}^{2}(G)$ is the small space of the bosonic model obtained by restricting the sum in (3.4) to the representations integrable at level $k-g^{\vee}$. Operators $Q_{0}^{ \pm}$preserve $\mathbb{H}_{0}^{\mathbf{R}}$ and reduce on it to the Dirac operators $\not_{ \pm}$of (4.23). As for the algebra $\mathcal{A}_{0}$, we shall choose it to be the small bosonic algebra acting trivially on the $W$ factor of $\mathbb{H}_{0}$. It is generated by the primary fields corresponding to states $|\phi\rangle \in \mathbb{H}_{0}^{\mathrm{NS}}$ sandwiched between the projectors on $\mathbb{H}_{0}^{\mathrm{R}}$. Clearly, the finite non-commutative geometry encoded by $\left(\mathbb{H}_{0}^{\mathrm{R}},\left.Q_{0}^{ \pm}\right|_{\mathbb{H}_{0}^{R}}, \mathcal{A}_{0}\right)$ is a deformation of the geodesic motion of the superparticle on $G$ to which it reduces in the limit $k \rightarrow \infty$.

For $\left(\mathbb{H}_{0},\left.Q_{0}^{ \pm}\right|_{\mathbb{H}_{0}}, \mathcal{A}_{0}\right)$, (non-commutative) 1-forms are $\sqrt{\frac{2}{k}} \sum_{j} a_{j 0}\left[J_{0}^{A \pm}, a_{j_{1}}\right] \psi_{0}^{A \pm}$, with $a_{j 0}$, $a_{j 1} \in \mathcal{A}_{0}$, and the question arises whether any operator $a^{A} \psi_{0}^{A \pm}, a^{A} \in \mathcal{A}_{0}$, may be cast in such a form. In other words, is the $\mathcal{A}_{0}$-module $\Omega^{1}\left(\mathcal{A}_{0}\right)$ free, with a basis given by $\left(\psi_{0}^{A \pm}\right)$ ? This appears to be a technically rather difficult question. The answer is yes for $G=S U(2)$ and $k-g^{\vee}=k-2 \leq 2$. If it is positive in general then one may define a connection $\nabla$ on $\Omega^{1}\left(\mathcal{A}_{0}\right)$ essentially by the same formula (4.35) as used in the commutative case of SUSY quantum mechanics on $G$ :

$$
\nabla \omega=\sqrt{\frac{2}{k}} \psi_{0}^{A \pm} \otimes\left[J_{0}^{A \pm}, \omega\right]
$$

Just as there, $\psi_{0}^{A \pm}$ would provide a parallel basis of $\Omega^{1}\left(\mathcal{A}_{0}\right)$ and $\nabla$ would have vanishing curvature: it appears that the effective target of the SUSY WZW model is a non-commutative space supplied with flat connections of non-zero torsion. Although this has been proven only 
in the limit $k=\infty$ and for $G=S U(2)$ at $k-2=1,2$, it is reasonable to expect that it is true more generally.

\subsection{SUSY coset mechanics}

Let us return to the motion of a superparticle on a group $G$, with $L^{2}(G) \otimes W$ giving the space of quantum states. Let $H$ be a connected subgroup of $G$. We shall extend the construction of the coset quantum mechanics to the SUSY case. The generators $J^{\text {at }}$ of leftright regular actions of $\mathbf{h} \subset \mathbf{g}$ on $L^{2}(G)$ define two commuting representations ${ }^{12}$ of $\mathbf{h}$ in $L^{2}(G) \otimes W$. By taking

$$
\widetilde{J}^{\mathrm{a} \pm} \equiv J^{\mathrm{a} \pm}+\frac{1}{2 i} f^{\mathrm{a} \beta \gamma} \psi^{\beta \pm} \psi^{\gamma \pm}
$$

one obtains another such pair of representations. As the space of states of the coset quantum mechanics, we shall take

$$
\left(L^{2}(G) \otimes W\right)_{H} \equiv\left\{|\phi\rangle \in L^{2}(G) \otimes W \quad\left(\psi^{\mathrm{a}+}+\psi^{\mathrm{a}-}\right)|\phi\rangle=0=\left(\widetilde{J}^{\mathrm{a}+}+\widetilde{J}^{\mathrm{a}-}\right)|\phi\rangle\right\} .
$$

Let

$$
\not_{ \pm}^{\prime}=\sqrt{\frac{2}{k}}\left(\psi^{\mathrm{a} \pm} \tilde{J}^{\mathrm{a} \pm}-\frac{i}{6} f^{\mathrm{abc}} \psi^{\mathrm{a} \pm} \psi^{\mathrm{b} \pm} \psi^{\mathrm{c} \pm}\right)
$$

Then

$$
\partial_{ \pm}^{\text {cs }} \equiv \partial_{ \pm}-\partial_{ \pm}^{\prime}=\sqrt{\frac{2}{k}}\left(\psi^{\alpha \pm} J^{\alpha \pm}-\frac{i}{6} f^{\alpha \beta \gamma} \psi^{\alpha \pm} \psi^{\beta \pm} \psi^{\gamma \pm}\right)
$$

commutes with $\psi^{\mathrm{a} \pm}$ and with $\widetilde{J}^{\mathrm{a} \pm}$ and defines the coset Dirac operators acting on $\left(L^{2}(G) \otimes\right.$ $W)_{H}$. Finally, the natural action of $C^{\infty}(G)_{H}$ on $L^{2}(G) \otimes W$ descends to $\left(L^{2}(G) \otimes W\right)_{H}$, so that we may regard

$$
\left(\left(L^{2}(G) \otimes W\right)_{H}, \not \partial_{ \pm}^{c s}, C^{\infty}(G)_{H}\right)
$$

as the triple encoding the geometry of the SUSY coset quantum mechanics.

Let

$$
\left.W^{\perp}=\left\{|\phi\rangle \in W\left|\left(\psi^{\mathbf{a}+}+\psi^{\mathbf{a}-}\right)\right| \phi\right\rangle=0\right\} .
$$

$W^{\perp}$ carries the (irreducible) representation of the algebra generated by $\psi^{\alpha \pm}$. Note that the generators $\tilde{J}^{\text {at }}$ of $\mathrm{h}$ may be restricted to $L^{2}(G) \otimes W^{\perp} .\left(L^{2}(G) \otimes W\right)_{H}$ is the subspace of $L^{2}(G) \otimes W^{\perp}$ invariant under the diagonal action of $H$ defined by $\left(\widetilde{J}^{a+}+\widetilde{J}^{a-}\right)$. This picture of SUSY coset quantum mechanics may be easily obtained by a quantization of the classical system given by the action functional

$$
S\left(g(\cdot), \psi^{\alpha \pm}(\cdot), A_{ \pm}(\cdot)\right)=-\frac{k}{4} \int \operatorname{tr}\left(g^{-1} \partial_{\tau} g\right)^{2} d \tau
$$

\footnotetext{
${ }^{12}$ recall that $a, b, \ldots$ label the generators of $\mathbf{h} \subset \mathbf{g}$ and $\alpha, \beta, \ldots$ the ones in the perpendicular subspace $\mathbf{h}^{\perp}$
} 


$$
\begin{aligned}
+\frac{k}{2} \int \operatorname{tr}\left(\left(g \partial_{\tau} g^{-1}\right) A_{-}+\right. & \left.A_{+}\left(g^{-1} \partial_{\tau} g\right)+g A_{+} g^{-1} A_{-}-A_{+} A_{-}\right) d \tau \\
+ & \frac{i}{2} \sum_{+,-} \int \psi^{\beta \pm}\left(\delta^{\beta \gamma} \partial_{t}-i f^{\mathrm{a} \beta \gamma} A_{\mp}^{\mathrm{a}}\right) \psi^{\gamma \pm} d \tau
\end{aligned}
$$

with the Grassmann variables $\psi^{\alpha \pm}(\tau)$ solely in the $\mathbf{h}^{\perp}$ directions and gauge fields $A_{ \pm} \equiv t^{\mathbf{a}} A_{ \pm}^{\mathrm{a}}$ with values in $h$. Elimination of $A_{ \pm}$from the action leads to the action for a superparticle moving on $G / \operatorname{Ad}(G)$ with the metric given by eqs. (3.28) or (3.29). Recall that the (co-)vectors tangent to $G / \operatorname{Ad}(H)$ could be parametrized by elements of $\mathbf{h}^{\perp}$ by formulae (3.32) or (3.32). These are the parametrizations which allow to view $t^{\alpha} \psi^{\alpha \pm}(\tau)$ as taking values in the spaces tangent to $G / \operatorname{Ad}(H)$. It is possible then to recover the torsion form from the effective action obtained by eliminating the gauge fields. It is given by the expression

$$
\frac{i k}{3} \operatorname{tr}\left(g^{-1} d g\right)^{\wedge 3}+i k d \operatorname{tr}\left(g^{-1} d g\right)\left(E-E \operatorname{Ad}_{g} E\right)^{-1}\left(g d g^{-1}\right)
$$

which defines a, possibly singular, closed 3 -form on $G / \operatorname{Ad}(H)$.

Because of the presence of dilaton, the coset quantum mechanics as defined by the triple (4.63) does not, however, coincide with the one obtained by quantization of the superparticle on $G / \operatorname{Ad}(H)$ with the metric and torsion form described above. It differs from those by ordering effects. They are rather complicated but the non-commutative formalism offers a possibility to study the coset geometry directly, using data (4.63). It is not hard to see, for example, that 1 -forms $\omega=\sum_{j} a_{j 0}\left[\not_{ \pm}, a_{j 1}\right]$ are arbitrary operators on $\left(L^{2}(G) \otimes W\right)_{H}$ of the form $a^{\alpha} \psi^{\alpha \pm}$, where $a^{\alpha}$ are functions on $G$ such that

$$
\left[J^{\mathrm{a}+}+J^{\mathrm{a}-}, a^{\alpha}\right]=i f^{\mathrm{a} \alpha \beta} a^{\beta} .
$$

We may identify $\psi^{\alpha \pm}$ with the standard 1 -forms on $G$ given by eqs. (3.31) and (3.32). Formula

$$
\nabla \omega=\sqrt{\frac{2}{k}} \psi^{\alpha \pm} \otimes\left[J^{\alpha \pm}, \omega\right]
$$

defines again a connection on the cotangent bundle $\Omega^{1}(\mathcal{A})$ which, however, has non-trivial curvature (also the Ricci and the scalar ones) and torsion.

\subsection{SUSY coset CFT}

The SUSY coset construction generalizes to the supersymmetric case [28][38]. Introducing in the Hilbert space of the SUSY WZW model based on the group $G$ the modified $\hat{\mathbf{h}} \subset \widehat{\mathbf{g}}$ currents

$$
\widetilde{J}_{n}^{\mathrm{a \pm}}=J_{n}^{\mathrm{a \pm}}+\frac{1}{2 i} f^{\mathrm{a} \beta \gamma} \sum_{m}: \psi_{m}^{\beta \pm} \psi_{n-m}^{\gamma \pm}:
$$

with central charge $k-h^{\vee}$, we may define the Hilbert space of the coset theory as

$$
\mathbb{H} \equiv \mathbb{H}^{N S} \oplus \mathbb{H}^{R}=\left\{\begin{array}{l|l}
|\phi\rangle \in \mathbb{H}_{\mathrm{wZW}} & \mid \begin{array}{l}
\left.\psi_{n}^{\mathrm{a} \pm}|\phi\rangle=0=\widetilde{J}_{n}^{\mathrm{a}}|\phi\rangle\right) \text { for } n>0, \\
\left(\psi_{0}^{\mathrm{a}+}+\psi_{0}^{\mathrm{a}-}\right)|\phi\rangle=0=\left(\widetilde{J}_{0}^{\mathrm{a}+}+\widetilde{J}_{0}^{\mathrm{a}-}\right)|\phi\rangle
\end{array}
\end{array}\right\}
$$


where $\mathbb{H}_{\text {wzw }}$ now denotes the space of states of the SUSY WZW model with group $G$. The operators

$$
L_{n}^{\mathrm{cs} \pm}=L_{\mathrm{bos} n}^{ \pm}+l_{n}^{ \pm}-L_{\text {bos } n}^{\prime \pm}-l_{n}^{\prime \pm}
$$

where

$$
\begin{aligned}
& L_{\text {bos } n}^{ \pm}=\frac{1}{k} \sum_{m}: J_{m}^{A \pm} J_{n-m}^{A \pm}:, \quad l_{n}^{ \pm}=-\sum_{m} \frac{1}{2} m: \psi_{m}^{A \pm} \psi_{n-m}^{A \pm}:\left(+\frac{3 \operatorname{dim}(G)}{48} \delta_{n, 0}\right), \\
& L_{\text {bos } n}^{\prime \pm}=\frac{1}{k} \sum_{m}: \tilde{J}_{m}^{a \pm} \tilde{J}_{n-m}^{a \pm}:, \quad l_{n}^{\prime \pm}=-\sum_{m} \frac{1}{2} m: \psi_{m}^{\mathrm{a \pm}} \psi_{n-m}^{\mathrm{a \pm}}:\left(+\frac{3 \operatorname{dim}(H)}{48} \delta_{n, 0}\right)
\end{aligned}
$$

preserve $\mathbb{H}$ and define on it an action of a commuting pair of Virasoro algebras with central charge $c_{\text {susY } k}^{G}-c_{\text {SUSY } k}^{H}$. Let

$$
Q_{n}^{\prime \pm}=\sqrt{\frac{2}{k}}\left(\sum_{m} \psi_{m}^{\mathrm{a} \pm} \widetilde{J}_{n-m}^{\mathrm{a} \pm}-\frac{i}{6} f^{\mathrm{abc}} \sum_{m, r}: \psi_{m}^{\mathrm{a} \pm} \psi_{r}^{\mathrm{b} \pm} \psi_{n-m-r}^{\mathrm{c \pm}}:\right)
$$

Then

$$
Q_{n}^{\mathrm{cs} \pm} \equiv Q_{n}^{ \pm}-Q_{n}^{\prime \pm}=\sqrt{\frac{2}{k}}\left(\sum_{m} \psi_{m}^{\alpha \pm} J_{n-m}^{\alpha \pm}-\frac{i}{6} f^{\alpha \beta \gamma} \sum_{m, r}: \psi_{m}^{\alpha \pm} \psi_{r}^{\beta \pm} \psi_{n-m-r}^{\gamma \pm}:\right)
$$

preserve $\mathbb{H}$ and extend the Virasoro algebras $\left(L_{n}^{\mathrm{cs} \pm}\right)$ to the super-Virasoro ones.

On the Lagrangian level, the SUSY coset models correspond to the action

$$
S\left(g, \psi^{\alpha \pm}, A_{ \pm}\right)=S_{\mathrm{bos}}\left(g, A_{ \pm}\right)+\frac{i}{4 \pi} \sum_{+,-} \int \psi^{\beta \pm}\left(\delta^{\beta \gamma} \partial_{\mp}-i f^{\mathrm{a} \beta \gamma} A_{ \pm}^{\mathrm{a}}\right) \psi^{\gamma \pm} d \sigma d \tau
$$

where $S_{\text {bos }}\left(g, A_{ \pm}\right)$is as in (3.40), but with $k \mapsto k-g_{:}^{\vee}, \psi^{ \pm}$take values in $\mathbf{h}^{\perp}$ and $A_{ \pm}$in $\mathbf{h}$.

Let $\mathbb{H}_{0}^{\mathbb{N S}}\left(\mathbb{H}_{0}^{\mathrm{R}}\right)$ denote the subspace in the Neveu-Schwarz (Ramond) sector of the coset theory annihilated by $\psi_{n}^{A \pm}$ and $J_{n}^{A \pm}$, for $n>0$. Notice that $\mathbb{H}_{0}^{\mathrm{NS}}$ may be naturally identified with $L_{k-g^{\vee}}^{2}(G)_{H}$, the subspace of states of the bosonic coset quantum mechanics corresponding to the integrable representations. Similarly $\mathbb{H}_{0}^{\mathrm{R}}$ is naturally isomorphic to $\left(L_{k-g^{\vee}}^{2}(G) \otimes W\right)_{H}$ i.e. to a subspace of the space of states of the SUSY coset quantum mechanics. The action of $Q_{0}^{\text {cst }}$ on $\mathbb{H}_{0}^{\mathrm{R}}$ reduces to that of $\phi_{ \pm}^{\mathrm{cs}}$. The (super-Virasoro) primary fields $V_{|\phi\rangle}^{\mathrm{cs}}\left(z_{ \pm}\right)$of the coset theory corresponding to vectors $|\phi\rangle \in \mathbb{H}^{\mathrm{NS}}$ annihilated by $Q_{n}^{\text {cs } \pm}$ and $L_{n}^{\text {cs } \pm}$ for $n>0$ may be used to generate the bosonic field algebra $\mathcal{A}$. Similarly, operators $\left.E_{0}^{\mathrm{R}} V_{|\phi\rangle}^{\mathrm{cs}}(1)\right|_{\mathbb{F}_{0}^{\mathrm{R}}}$ with $|\phi\rangle \in \mathbb{H}_{0}^{\mathrm{NS}}$, where $E_{0}^{\mathrm{R}}$ is the orthogonal projection on $\mathbb{H}_{0}^{\mathrm{R}}$, generate a small algebra $\mathcal{A}_{0}$. The triple $\left(\mathbb{H}^{\mathrm{R}}, Q_{0}^{\mathrm{cs} \pm}, \mathcal{A}\right)$ represents the geometry of the SUSY coset CFT, whereas $\left(\mathbb{H}_{0}^{\mathrm{R}},\left.Q_{0}^{\mathrm{cs} \pm}\right|_{\mathbb{H}_{0}^{\mathrm{R}}}, \mathcal{A}_{0}\right)$ encodes that of its effective target. The latter is a finite non-commutative deformation of the geometry of the SUSY coset quantum mechanics discussed above.

As in the bosonic case, one may alternatively define an effective target geometry of the SUSY coset models using the SUSY extension of the Virasoro algebra. This would give the triple $\left(\mathbb{H}_{0}^{\prime R},\left.Q_{0}^{\text {cs士 }}\right|_{\mathbb{H}_{0}^{\prime}}, \mathcal{A}_{0}^{\prime}\right)$, where

$$
\left.\mathbb{H}_{0}^{\mathrm{NS}, \mathrm{R}}=\left\{|\phi\rangle \in \mathbb{H}^{\mathrm{NS}, \mathrm{R}}\left|Q_{n}^{\mathrm{cs} \pm}\right| \phi\right\rangle=0=L_{n}^{\mathrm{cs} \pm}|\phi\rangle, \text { for } n>0,\left(L_{0}^{\mathrm{cs}+}-L_{0}^{\mathrm{cs}-}\right)|\phi\rangle=0\right\}
$$


and $\mathcal{A}_{0}^{\prime}$ is generated by sandwiching the primary fields $V_{|\phi\rangle}^{\mathrm{cs}}(1)$ corresponding to vectors $|\phi\rangle \in$ $\mathbb{H}_{0}^{\prime N S}$ between orthogonal projections onto $\mathbb{H}_{0}^{\prime R}$. The above construction of the effective target is consistent for any $\mathrm{N}=1$ super-CFT.

An interesting open problem is to find natural connections associated with the coset targets and to study their flatness properties. In the quantum mechanical case the coset geometry corresponds to a dilatonic deformation of Riemannian geometry. The presence of the dilaton changes the perturbative conditions for the conformality of the sigma models [7] so that the presence of non-trivial Ricci curvature in the coset target should not be surprising. One may expect a similar deformation to occur for the non-commutative effective targets of the coset models of field theory defined above.

\section{5. $\mathrm{N}=2$ CFT and mirror symmetry}

\section{1. $\mathrm{N}=2$ SUSY coset models}

In [40][41], Kazama and Suzuki pointed out that there is a class of SUSY $G / H$ coset models which possess extended $\mathrm{N}=2$ superconformal symmetry. The latter requires two series of operators $G_{n}^{ \pm}$and $\bar{G}_{n}^{ \pm}$extending the Virasoro algebras $\left(L_{n}^{ \pm}\right)$and a commuting pair of $u(1)$ current algebras $\left(j_{n}^{ \pm}\right)$with the commutation relations

$$
\begin{aligned}
& {\left[j_{n}^{ \pm}, j_{m}^{ \pm}\right]=\frac{1}{3} c n \delta_{n+m, 0}} \\
& {\left[j_{n}^{ \pm}, G_{m}^{ \pm}\right]=G_{n+m}^{ \pm},} \\
& {\left[j_{n}^{ \pm}, \bar{G}_{m}^{ \pm}\right]=-\bar{G}_{n+m}^{ \pm}} \\
& {\left[L_{n}^{ \pm}, G_{m}^{ \pm}\right]=\left(\frac{n}{2}-m\right) G_{n+m}^{ \pm}} \\
& {\left[L_{n}^{ \pm}, \bar{G}_{m}^{ \pm}\right]=\left(\frac{n}{2}-m\right) \bar{G}_{n+m}^{ \pm},} \\
& \left\{G_{n}^{ \pm}, \bar{G}_{m}^{ \pm}\right\}=2 L_{n+m}^{ \pm}+(n-m) j_{n+m}^{ \pm}+\frac{1}{3} c\left(n^{2}-\frac{1}{4}\right) \delta_{n+m, 0} .
\end{aligned}
$$

The operators $G_{n}^{ \pm}$commute with $L_{m}^{\mp}$ and $j_{m}^{\mp}$ and anticommute with $G_{m}^{\mp}$ and $\bar{G}_{m}^{\mp}$. The N=2 superconformal symmetry implies the $\mathrm{N}=1$ one: we may set $Q_{n}^{ \pm}=\frac{1}{\sqrt{2}}\left(G_{n}^{ \pm}+\bar{G}_{n}^{ \pm}\right)$. One may realize the $\mathrm{N}=2$ algebra in SUSY coset models if $G / H$ is a homogeneous Kähler manifold, in particular, if $G / H$ is a hermitian symmetric space. In the latter situation, one may decompose

$$
\left(\mathbf{h}^{\perp}\right)^{\mathbb{C}}=\mathbf{t} \oplus \overline{\mathbf{t}}
$$

where $\mathbf{t}$ and $\overline{\mathbf{t}}$ are complex conjugate abelian Lie subalgebras of $\mathbf{g}$, preserved $\left(\bmod \mathbf{h}^{\mathbb{C}}\right)$ by the adjoint action of $\mathbf{h}$, isotropic for $\operatorname{tr}$ and s.t. $[\mathbf{t}, \overline{\mathbf{t}}] \subset \mathbf{h}$. We shall choose a basis $\left(t^{\alpha}, t^{\bar{\alpha}}\right)$ of $\left(\mathbf{h}^{\perp}\right)^{\mathbb{C}}$ with the property that $t^{\bar{\alpha}}=\overline{t^{\alpha}}$ and that $\operatorname{tr} t^{\alpha} t^{\tilde{\beta}}=\frac{1}{2} \delta^{\alpha \beta}$. Then

$$
\begin{aligned}
G_{n}^{ \pm} & =\frac{2}{\sqrt{k}} \sum_{m} \psi_{m}^{\alpha} J_{n-m}^{\bar{\alpha}}, \\
\bar{G}_{n}^{ \pm} & =\frac{2}{\sqrt{k}} \sum_{m}^{m} \psi_{m}^{\bar{\alpha}} J_{n-m}^{\alpha},
\end{aligned}
$$




$$
j_{n}^{ \pm}=\frac{k-g^{\vee}}{k} \sum_{m}: \psi_{m}^{\alpha \pm} \psi_{n-m}^{\bar{\alpha} \pm}:-\frac{2 i}{k} f^{\mathbf{a} \alpha \bar{\alpha}} J_{n}^{\mathbf{a} \pm}
$$

The simplest hermitian symmetric space is $P \mathbb{C}^{\mathbf{1}}=S U(2) / U(1)$. It gives the minimal $\mathrm{N}=2$ series of the SUSY coset theories with central charges $\frac{3(k-2)}{k}$. The space of states of these models is a finite sesquilinear combination of irreducible unitary representations of the $\mathrm{N}=2$ superconformal algebra (with $c<3$ ) [6]. Another example of a hermitian symmetric space is provided by the Grassmannian $S U(n+m) / S U(n) \times S U(m) \times U(1)$. It leads to a series of $\mathrm{N}=2$ coset models with central charges $\frac{3 n m(k-n-m)}{k}$. The complete list of (compact) hermitian symmetric spaces is short [32] and contains, besides the above examples, still $S O(2 n) / S O(n) \times$ $S O(2), S O(2 n) / S U(n) \times U(1), S P(n) / S U(n) \times U(1), E_{6} / S O(10) \times U(1)$ and $E_{7} / E_{6} \times U(1)$.

We may identify the effective target geometry, $\left(\mathbb{H}_{0}^{\mathrm{R}},\left.Q_{0}^{\mathrm{cs} \pm}\right|_{\mathbb{H}_{0}^{R}}, \mathcal{A}_{0}\right)$, of $\mathrm{N}=2$ SUSY coset models in the same way as for the $\mathrm{N}=1$ ones. Alternatively, we may pose

$$
\left.\mathbb{H}^{\prime N S, R}=\left\{|\phi\rangle \in \mathbb{H}^{\mathrm{NS}, \mathrm{R}}\left|L_{n}^{ \pm}, G_{n}^{ \pm}, \bar{G}_{n}^{ \pm}, j_{n}^{ \pm}\right| \phi\right\rangle \underset{n>0}{=} 0,\left(L_{0}^{+}-L_{0}^{-}\right)|\phi\rangle=0\right\}
$$

and define the small algebra $\mathcal{A}_{0}^{\prime}$ to be the one generated by the primary fields $V_{|\phi\rangle}(1),|\phi\rangle \in$ $\mathbb{H}^{\prime}{ }_{0}^{\mathrm{NS}}$, multiplied by the orthogonal projection onto $\mathbb{H}_{0}^{\prime \mathrm{R}}$ from both sides ${ }^{13}$. The latter construction may be done for any $\mathrm{N}=2$ conformal model. Notice that $\mathbb{H}_{0}^{\mathrm{R}}\left(\mathbb{H}_{0}^{\prime}\right)$ is preserved by the operators $G_{0}^{ \pm}, \bar{G}_{0}^{ \pm}$and $j_{0}^{ \pm}$which, when restricted to $\mathbb{H}_{0}^{\mathrm{R}}\left(\mathbb{H}_{0}^{\prime \mathbf{R}}\right)$, satisfy the algebra:

$$
\begin{aligned}
& \left(G_{0}^{ \pm}\right)^{2}=0=\left(\bar{G}_{0}^{ \pm}\right)^{2}, \quad\left\{G_{0}^{+}, \bar{G}_{0}^{+}\right\}=\left\{G_{0}^{-}, \bar{G}_{0}^{-}\right\}, \\
& {\left[j_{0}^{ \pm}, G_{0}^{ \pm}\right]=G_{0}^{ \pm},\left[j_{0}^{ \pm}, \bar{G}_{0}^{ \pm}\right]=-\bar{G}_{0}^{ \pm},\left(G_{0}^{ \pm}\right)^{*}=\bar{G}_{0}^{ \pm},\left(j_{0}^{ \pm}\right)^{*}=j_{0}^{ \pm} \text {. }}
\end{aligned}
$$

The operators with superscript + (anti)commute with the ones with superscript - . This is the same algebra as the one satisfied by operators $G^{ \pm} \equiv \Gamma^{\alpha \pm} \nabla_{\alpha}, \bar{G}^{ \pm} \equiv \Gamma^{\bar{\alpha} \pm} \nabla_{\bar{\alpha}}$ and $j^{ \pm} \equiv \frac{1}{4} \gamma_{\alpha \bar{\beta}}\left[\Gamma^{\bar{\beta} \pm}, \Gamma^{\alpha \pm}\right]$ acting on sections of the bundle $S \otimes S$ (or $S \otimes S \otimes \mathbb{C}^{2}$ ) over a Kähler manifold $M$ with the Kähler metric $\gamma_{\alpha \bar{\beta}} d z^{\alpha} d \overline{z^{\beta}}$ and the metric connection $\nabla$. Equivalently, we may consider the operators $\partial, \bar{\partial}^{*}, \partial^{*}, \bar{\partial}$ on $L^{2}(\Lambda M)$ and interpret $\pm j^{ \pm}$as counting the degrees in $\wedge^{p, q} M$. Since $\left(G_{0}^{ \pm}\right)^{2}=0=\left(\bar{G}_{0}^{ \pm}\right)^{2}$, we may consider the cohomology of any of those operators. In fact, all these cohomologies coincide and may be identified with the subspace of $\mathbb{H}^{\mathrm{R}}$ annihilated by $G_{0}^{ \pm}$and $\bar{G}_{0}^{ \pm}$. By analogy to the situation in the Kähler geometry, we shall call this subspace "harmonic" and we shall denote it by $\mathbb{H}^{\text {harm }}$. It is easy to see that $\mathbb{H}^{\text {harm }} \subset \mathbb{H}^{\prime \mathbf{R}}$. It coincides with the space of Ramond sector ground states corresponding to the eigenvalue $\frac{c}{24}$ of $L_{0}^{ \pm}$.

In the classical case, the space of harmonic forms on a Kähler manifold $M$ may be identified with the de Rham cohomology ring of $M$. Does the ring structure have a counterpart in $\mathbb{H}^{\text {harm }}$ ? In $\mathrm{N}=2$ theory (with integral $j_{0}+\bar{j}_{0}$ charges), there exists a unitary transformation $U$ from $\mathbb{H}^{\mathrm{R}}$ to $\mathbb{H}^{\mathrm{NS}}$ (the spectral flow [46]) s.t.

$$
U^{-1}\left(L_{n}^{ \pm} \mp \frac{1}{2} j_{n}^{ \pm}\right) U=L_{n}^{ \pm}-\frac{c}{24} \delta_{n, 0}
$$

\footnotetext{
${ }^{13}$ it is also natural to require here that $j_{0}^{+}|\phi\rangle=j_{0}^{-}|\phi\rangle=0$
} 


$$
\begin{array}{ll}
U^{-1} j_{n}^{ \pm} U & =j_{n}^{ \pm} \pm \frac{c}{6} \delta_{n, 0}, \\
U^{-1} G_{n \mp 1 / 2}^{ \pm} U & =G_{n}^{ \pm}, \\
U^{-1} \bar{G}_{n \pm 1 / 2}^{ \pm} U & =\bar{G}_{n}^{ \pm} .
\end{array}
$$

$U$ maps $\mathbb{H}^{\text {harm }}$ onto the so called chiral-chiral primary subspace $\mathbb{H}^{(c, c)}$ of $\mathbb{H}^{\mathrm{NS}}$ annihilated by $G_{\mp 1 / 2}^{ \pm}$and $\bar{G}_{ \pm 1 / 2}^{ \pm}$. The primary fields $V_{|\phi\rangle}\left(z^{ \pm}\right)$corresponding to $|\phi\rangle \in \mathbb{H}^{(c, c)}$ have non-singular operator product, i.e. they may be multiplied point-wise. Moreover, their product is the vertex operator of another (possibly vanishing) state in $\mathbb{H}^{(c, c)}$. This way $\mathbb{H}^{(c, c)}$ becomes a gradedcommutative "chiral-chiral" ring [42]. By the action of $U$, the ring structure may be carried over to $\mathbb{H}^{\text {harm }}$. We may then think about the data

$$
\left(\mathbb{H}_{0}^{\mathrm{R}}, G_{0}^{+}, \bar{G}_{0}^{-}, j_{0}^{ \pm}, \mathcal{A}_{0}\right) \quad \text { or }\left(\mathbb{H}^{\prime}{ }_{0}^{\mathrm{R}}, G_{0}^{+}, \bar{G}_{0}^{-}, j_{0}^{ \pm}, \mathcal{A}_{0}^{\prime}\right) \quad \text { and } \quad \mathbb{H}^{(\mathrm{c}, \mathrm{c})}
$$

as representing the effective target geometry and cohomology of the $\mathrm{N}=2 \mathrm{CFT}$, a deformation of the Kähler geometry $\left(L^{2}(\wedge M), \partial, \bar{\partial}, j^{ \pm}, C^{\infty}(M)\right)$ and of its de Rham cohomology.

It has been shown in [25][26] that for certain orbifolds of the tensor products of minimal $\mathrm{N}=2$ models which have integral $j_{0}^{ \pm}$charges, the rings $\mathbb{H}^{(\mathrm{c}, \mathrm{c})}$ are deformations of the de Rham cohomology rings of certain Calabi-Yau manifolds ${ }^{14}$. In particular, the dimensions of the spaces of harmonic states with $j_{0}^{ \pm}$charges $p-\frac{c}{6}, \frac{c}{6}-q$ are equal to the Hodge numbers ${ }^{15} h_{M}^{p, q}$ of a Calabi-Yau space $M$ of complex dimension $d=\frac{c}{3}$ ). One may also argue directly (see E. Witten's contribution to [55]) that, for sigma models with Calabi-Yau targets, the chiral-chiral ring is the cohomology ring of the manifold deformed by instanton effects ${ }^{16}$. The deformation disappears in the semi-classical limit sending the radius of the target space to infinity. One should expect that, in an appropriate classical limit, also the effective target geometry of the $\mathrm{N}=2$ models, not only their cohomology, becomes that of Calabi-Yau manifolds or of their torsion or/and dilatonic versions, see M. Roček's contribution to [55]. This issue deserves further study. It should be remarked, that a complex version of non-commutative geometry, appropriate for study of data (5.7), still remains to be developed.

\subsection{Mirror targets}

The $N=2$ super-conformal algebra does not change if we reverse the sign of the $u(1)$ current $j_{n}$ interchanging at the same time $G_{n}$ and $\bar{G}_{n}$. Constructing the effective target geometry and cohomology in a given $\mathrm{N}=2$ model with integral $j_{0}^{ \pm}$charges after a replacement $j_{n}^{-} \mapsto$ $-j_{n}^{-}, G_{n}^{-} \leftrightarrow \bar{G}_{n}^{-}$, one obtains different objects: mirror effective target and the chiral-antichiral ring:

$$
\left(\mathbb{H}_{0}^{\mathrm{R}}, G_{0}^{+}, G_{0}^{-}, \pm j_{0}^{ \pm}, \mathcal{A}_{0}\right) \quad \text { or } \quad\left(\mathbb{H}_{0}^{\prime \mathrm{R}}, G_{0}^{+}, G_{0}^{-}, \pm j_{0}^{ \pm}, \mathcal{A}_{0}^{\prime}\right) \quad \text { and } \quad \mathbb{H}^{(\mathrm{c}, \mathrm{a})} .
$$

It has been checked that in some cases $\mathbb{H}^{(\mathbf{c}, \mathbf{a})}$ is a deformed cohomology ring of a different Calabi-Yau manifold $\widetilde{M}$ with the Hodge numbers

$$
h_{\tilde{M}}^{p, q}=h_{M}^{p, d-q} .
$$

\footnotetext{
${ }^{14}$ i.e. Kähler manifolds with SU(3) holonomy and, consequently, Ricci flat

15 i.e. to the dimensions of the spaces of harmonic $(p, q)$-forms

${ }^{16}$ instantons of the sigma model with Kähler target $M$ are complex curves in $M$
} 
Besides, the chiral-chiral ring $\mathbb{H}^{(c, c)}$ should then coincide with the undeformed Dolbeault cohomology ring with values in the exterior algebra of the holomorphic tangent bundle of $\widetilde{M}$. The latter is usually easier to compute. This way, one may extract non-trivial information about instanton numbers for a Calabi-Yau manifold $M$, usually hard to obtain directly, from an easy calculation of the Dolbeault cohomology on the mirror image $\widetilde{M}$ of $M$ [2][5]. The mirror symmetry $M \longleftrightarrow \widetilde{M}$ should extend from special pairs, where it was directly verified, to the moduli space of Calabi-Yau spaces, with the interchange of the role of moduli of complex structures (counted by $h^{d-1,1}=h^{1, d-1}$ ) and of Kähler structures (corresponding to $h^{1,1}$ ).

The $\mathrm{N}=2$ conformal models are difficult to control out of special points but the cohomological information given by the chiral-chiral or chiral-antichiral rings is contained in the topological field theories, easier to solve, obtained by coupling the $u(1)$ currents of the conformal models to the spin connection [53][18]. The chiral-chiral and chiral-antichiral rings reduce to the de Rham cohomology rings of Calabi-Yau spaces $M$ and $\widetilde{M}$ in different semi-classical limits. Although the geometry of the complete CFT is more difficult to control then its cohomology, the construction which associates to a given $\mathrm{N}=2$ theory a mirror pair of effective (non-commutative) targets

$$
\left(\mathbb{H}_{0}^{\prime \mathrm{R}}, G_{0}^{+}, \bar{G}_{0}^{-}, j_{0}^{ \pm}, \mathcal{A}_{0}^{\prime}\right) \quad \text { and } \quad\left(\mathbb{H}_{0}^{\prime \mathrm{R}}, G_{0}^{+}, G_{0}^{-}, \pm j_{0}^{ \pm}, \mathcal{A}_{0}^{\prime}\right)
$$

is essentially tautological: non-commutative geometry in its complex version should provide a natural setup for the mirror symmetry.

The simplest example of a mirror pair of Calabi-Yau spaces is obtained by taking the complex three-dimensional smooth projective variety $M$ defined by the equation

$$
z_{1}^{5}+z_{2}^{5}+z_{3}^{5}+z_{4}^{5}+z_{5}^{5}=0
$$

in $P \mathbb{C}^{4}$ [30]. Its canonical bundle has vanishing first Chern class and, by the theorem of Yau proving Calabi's conjecture, it admits a metric with $S U(3)$ holonomy (unique up to normalization). The corresponding CFT is a projected version of the product of five copies of the minimal $k=5 \mathrm{~N}=2$ models. The mirror image $\widetilde{M}$ of $M$ is the orbifold ${ }^{17}$ of $M$ under the action of the $\mathbb{Z}_{5}^{3}$ group generated by

$$
\begin{aligned}
& \left(z_{1}, z_{2}, z_{3}, z_{4}, z_{5}\right) \mapsto\left(z_{1}, \rho z_{2}, \rho^{2} z_{3}, \rho^{3} z_{4}, \rho^{4} z_{5}\right) \\
& \left(z_{1}, z_{2}, z_{3}, z_{4}, z_{5}\right) \mapsto\left(z_{1}, \rho z_{2}, \rho z_{3}, \rho^{4} z_{4}, \rho^{4} z_{5}\right) \\
& \left(z_{1}, z_{2}, z_{3}, z_{4}, z_{5}\right) \mapsto\left(z_{1}, z_{2}, z_{3}, \rho z_{4}, \rho^{4} z_{5}\right)
\end{aligned}
$$

where $\rho$ is a fifth root of 1 . The relevant Hodge numbers are $h_{M}^{1,1}=h_{\tilde{M}}^{2,1}=1, h_{M}^{2,1}=h_{\tilde{M}}^{1,1}=$ 101. Many other examples of mirror pairs of Calabi-Yau spaces were explicitly identified, see $[30][9][55]$, and the subject is under intensive study [45][8][10] [33][5][1].

\footnotetext{
${ }^{17}$ more exactly, after the resolution of its singularities
} 


\section{References}

[1] Aspinwall, P. S., Greene, B. R., Morrison, D. R.: Calabi-Yau moduli space, mirror manifolds and spacetime topology change in string theory. hep-th/9309097

[2] Aspinwall, P. S., Morrison, D. R.: Topological field theory and rational curves. Oxford preprint OU-91-32p

[3] K. Bardakci, E. Rabinovici, B. Säring, String models with $c<1$ components. Nucl. Phys. B299, 151-182 (1988)

[4] Belavin A. A., Polyakov A. M., Zamolodchikov A. B.: Infinite conformal symmetry in two-dimensional quantum field theory. Nucl. Phys. B241, 333-380 (1984)

[5] Bershadsky, M., Cecotti, S., Ooguri, H., Vafa, C.: Holomorphic anomalies in topological field theories. Harvard Preprint HUTP-93/A008, hep-th/9302103

[6] Boucher, W., Friedan, D., Kent, A.: Determinant formulae and unitarity for the $N=2$ superconformal algebra in two dimensions, or exact results on string compactification. Phys. Let. B172, 316-322 (1986)

[7] Callan, C. G., Friedan, D., Martinec, E. J., Perry, M. J.: Strings in background fields. Nucl. Phys. B262, 593-609 (1985)

[8] Candelas, P., Derrick, E., Parkes, L.: Generalized Calabi-Yau manifolds and the mirror of a rigid manifold. hep-th/9304045

[9] Candelas, P., Lynker, M., Schimmrigk, R.: Calabi-Yau manifolds in weighted $\mathbb{P}_{4}$. Nucl. Phys. B341, 383-402 (1980)

[10] Candelas, P., de la Ossa, X., Font, A., Katz, Sh., Morrison, D. R.: Mirror symmetry for two parameter models $-I$. hep-th $/ 9308083$

[11] Chamseddine, A. H., Felder, G., Fröhlich, J.: Gravity in non-commutative geometry. ETH-TH/1992-44 preprint

[12] Connes, A.: Non commutative differential geometry, Publ. I.H.E.S. 62, 41-144 (1985)

[13] Connes, A.: Non Commutative Geometry. IHES/M/93/12 preprint, to be published by Academic Press

[14] Connes, A.: Non commutative geometry and physics. IHES/M/93/32 preprint

[15] Connes, A., Lott, J.: The metric aspect of non commutative geometry. In: New Symmetry Principles in Quantum Field Theory, eds. Fröhlich, J., 't Hooft, G., Jaffe, A., Mack, G., Mitter, P. K., Stora, R., Plenum Press, New York 1992, pp. 53-94

[16] Dijkgraaf, R., Verlinde, E., Verlinde, H.: String propagation in black hole geometry. Nucl. Phys. B371, 269-314 (1992) 
[17] Dubois-Violette, M., Kerner, R., Madore, J.: Non-commutative differential geometry of matrix algebras. J. Math. Phys. 31, 316-322 (1990)

[18] Eguchi, T., Yang, S.-K.: N=2 superconformal models as topological field theories. Mod. Phys. Lett. A5, 1693-1701 (1990)

[19] Friedan, D.: Nonlinear models in $2+\epsilon$ dimensions. Ann. Phys. 163, 318-419 (1985)

[20] Friedan, D., Qiu, Z., Shenker, S.: Conformal invariance, unitarity and critical exponents in two dimensions. Phys. Rev. Lett. 52, 1575-1578 (1984)

[21] Gawędzki, K.: Yang-Mills as Schrödinger quantum mechanics on the space of gauge group orbits. Phys. Rev. D26, 3593-3610 (1982)

[22] Gawędzki, K.: Non-compact WZW conformal field theories. IHES/P/91/73 preprint. In: New Symmetry Principles in Quantum Field Theory, eds. Fröhlich, J., 't Hooft, G., Jaffe, A., Mack, G., Mitter, P. K., Stora, R., Plenum Press, New York 1992, pp. 247- 274

[23] Gawȩdzki, K., Kupiainen, A.: G/H conformal field theory from gauged WZW model. Phys. Lett. B215, 119-123 (1988)

[24] Gawędzki, K., Kupiainen, A.: Coset construction from functional integrals. Nucl. Phys. B320, 625-668 (1989)

[25] Gepner, D.: Exactly solvable string compactifications on manifolds with $S U(N)$ holonomy. Phys. Lett. B199, 380-388 (1987)

[26] Gepner, D.: Yukawa couplings for Calabi-Yau string compactification. Nucl. Phys. B311, 191-204 (1988/89)

[27] Glimm, J., Jaffe, A.: Quantum Physics. A Functional Integral Point of View. Springer, Berlin 1987

[28] P. Goddard, A. Kent, D. Olive, Unitary representations of the Virasoro and super-Virasoro algebras. Commun. Math. Phys. 103, 105-119 (1986)

[29] Guillemin V., Sternberg, Sh.: Symplectic Techniques in Physics. Cambridge, Cambridge University Press 1984.

[30] Green, B. R., Plesser, M. R.: Duality in Calabi-Yau moduli space. Nucl. Phys. B338, $15-37(1990)$

[31] Green, M. B., Schwarz, J. H., Brink, L.: N=4 Yang-Mills and $N=8$ supergravity as limits of string theories. Nucl. Phys. B198, 474-492 (1982)

[32] S. Helgason, Differential Geometry, Lie Groups and Symmetric Spaces. Academic Press, New York 1978 
[33] Hosono, S., Klemm, A., Theisen, S., Yau, S.-T.: Mirror symmetry, mirror map and applications to Calabi-Yau hypersurfaces. hep-th/9308122

[34] Jaffe, A., Leśniewski, A.: Supersymmetric quantum fields and infinite dimensional analysis. In: Nonperturbative Quantum Field Theory. Eds. 't Hooft, G., Jaffe, A., Mack, G., Mitter, P. K., Stora, R., Plenum Press, New York 1988, pp. 227-280

[35] Jaffe, A., Leśniewski, A, Osterwalder, K.: Quantum K-theory: the Chern character. Commun. Math. Phys. 118, 1-14 (1988)

[36] Kac, M.: Can one hear the shape of a drum? Amer. Math. Monthly 73, 1-23 (1966)

[37] Kac, V. G.: Infinite Dimensional Lie Algebras. Cambridge University Press, Cambridge 1985

[38] Kac, V. G., Todorov, I. T.: Superconformal current algebras and their Unitary representations. Commun. Math. Phys. 102, 337-347 (1985)

[39] D. Karabali, Q. Park, H. J. Schnitzer, Z. Yang, A GKO construction based on a path integral formulation of gauged Wess-Zumino-Witten actions. Phys. Lett. B216, 307-312 (1989)

[40] Y. Kazama, H. Suzuki, New N=2 Supeconformal Field Theories and Superstring Compactification. Nucl. Phys. B321, 232-268 (1989)

[41] Y. Kazama, H. Suzuki, Characterization of N=2 Supeconformal Models Generated by the Coset Space Method. Phys. Lett. B216, 112-116 (1989)

[42] Lerche, W., Vafa, C., Warner, N. P.: Chiral rings in $\mathrm{N}=2$ superconformal theories. Nucl. Phys. B324, 427-474 (1989)

[43] Milnor, J.: Eigenvalues of the Laplace operator on certain manifolds. Proc. Nat. Acad. Sci. USA 51, 542 (1964)

[44] Polyakov, A. M.: Gauge Fields and Strings. Harwood Publishers 1987

[45] Schimmrigk, R.: Critical superstring vacua from noncritical manifolds: a novel framework for string compactification and mirror symmetry. Phys. Rev. Lett. 70, 3688-3691 (1993)

[46] Seiberg, N., Schwimmer, A.: Comments on the $\mathrm{N}=2,3,4$ superconformal algebras in two dimensions. Phys. Lett. B184, 191-196 (1987)

[47] Sugawara, H.: A field theory of currents. Phys. Rev. 170, 1659-1662 (1968)

[48] Verlinde, E.: Fusion rules and modular transformations in 2-d conformal field theory. Nucl. Phys. B300, 360-376 (1988)

[49] West, P.: Introduction to Supersymmetry and Supergravity, Extended Second Edition. World Scientific, Singapore 1990 
[50] Witten, E.: Constraints on supersymmetry breaking, Nucl. Phys. B202, 253-316 (1982)

[51] Witten, E.: Non-abelian bosonization in two dimensions. Commun. Math. Phys. 92, 455472 (1984)

[52] Witten, E.: Noncommutative geometry and string field theory, Nucl. Phys. B268, 253-294 (1986)

[53] Witten, E.: Topological sigma models. Commun. Math. Phys. 118, 411-449 (1988)

[54] Witten, E.: String theory and black holes. Phys. Rev. D 44, 314-324 (1991)

[55] Essays on Mirror Symmetry. Ed. S.-T. Yau, International Press, Hong Kong 1992

[56] Zamolodchikov, A. B.: Conformal symmetry and multicritical points in two-dimensional quantum field theory. Yad. Fiz. 44, $821-827$ (1986)

[57] Zamolodchikov, A. B.: Renormalization group and perturbation theory near fixed points in two-dimensional field theory. Yad. Fiz. 46, 1819-1831 (1987)

[58] Zamolodchikov, A. B., Fateev, V. A.: Operator algebra and correlation functions of the two-dimensional Wess-Zumino $S U(2) \times S U(2)$ chiral model. Yad. Fiz. 43, 1031-1044 (1986) 

